\title{
Comparative Analysis of Diatom Algae Diversity in the Pamir Protected Lakes, Tajikistan
}

\author{
S. Barinova ${ }^{1 *}$, T. Niyatbekov ${ }^{2}$ \\ ${ }^{* 1}$ Institute of Evolution, University of Haifa, Haifa (Israel) \\ ${ }^{2}$ Institute of Botany, Plant Physiology and Genetics, Dushanbe (Tajikistan)
}

*Corresponding Author: S. Barinova, Institute of Evolution, University of Haifa, Haifa (Israel)

\begin{abstract}
Diatom algae from six Pamir lakes located at an altitude from 3,729 to 4,213 $\mathrm{m}$ above the sea level were studied in 466 samples collected in summer period of 2000-2018. Altogether 300 species of diatom algae (330 with infraspecies) of 76 genera were revealed in periphytonic 466. The richest communities were found in two freshwater lakes Turumtaykul with 171 species (184 taxa) and Rangkul with 162 species (176 taxa). Floristic analysis with help of statistical methods show that the water salinity can be regulating factor of diatom diversity development in high mountain lakes. The Pinnularia genus was the richest in five from six lakes floras. The two floristic core were revealed in the analysis of the species overlapping. The natural status of the protected high mountain lakes as a result of our analysis is indicates a high level of conservation of diversity in the territory of the Tajik National Park and the adjacent high-mountainous areas. Advanced statistical methods help us to characterize the stability of studied algal floras in protected high mountain lakes as higher on the genera level then on the species level, and therefore species of studied floras can be source of evolutionary speciation.
\end{abstract}

Keywords: diatom algae, diversity, Pamir Natural Reserve, Tajikistan

\section{INTRODUCTION}

Pamir is one of high altitude area in Eurasia with close relations to Hindu Cush, Altay, and Himalayas. Numbers of lakes are placed in its territory, which are occupied by diverse algal communities. Algal species richness and occurrence up to now are studied not enough in mountain lakes because their usually placed in hard-to-reach areas. Most of natural lakes in Tajikistan are under protection [1] and can represent some natural environment as reference characteristics in the context of an increase in the anthropogenic load of the surrounding areas and climate changes. More of them, most number of the lakes take place in the Eastern Pamir Mountains and has been included to the Tajik Natural Reserve [2,3]. Despite the fact that in recent years we have been making efforts to enrich and summarize data on the Pamir algae, the study of algal diversity in Pamir lakes is in initial stage [4-9]. Although it's algal species richness and ecology can help to characterize the reference natural habitats in this important and interesting area of the world. In the protected lakes, waters a special community of algae with a specific species composition was formed which can characterize native diversity of algae in the high mountains of Central Asia.

The aim of this work were characteristic of taxonomical diversity and comparative floristic of the diatom algal flora of six lakes located in the Tajik National Park and surrounding high mountain areas..

\section{MATERIAL AND MethodS}

\subsection{Description of Study Site}

The Tajik National Park (TNP) is the biggest high mountain park and nature reserve in eastern Tajikistan with a total area of 2.6 million hectares, which is $18 \%$ of the total size of Tajikistan [2] (Fig. 1). It was established in 1992 and designated by Decision No. 267 of the Tajikistan Government. In 2013, the park was accepted as UNESCO World Heritage.

The national park features a mix of steppe, desert, grassland and alpine regions. The climatic features of this territory (which also called "the World Roof") are a long cold winters and cool summers, with 
an average annual rainfall of $12.7 \mathrm{~cm}$ only [3]. The lakes in Pamir Mountains has a wide range of dissolved solids in water and are affiliated to three geological formations: Hydrocarbonate, Sulfate, and Sodium (Table 1) [10].

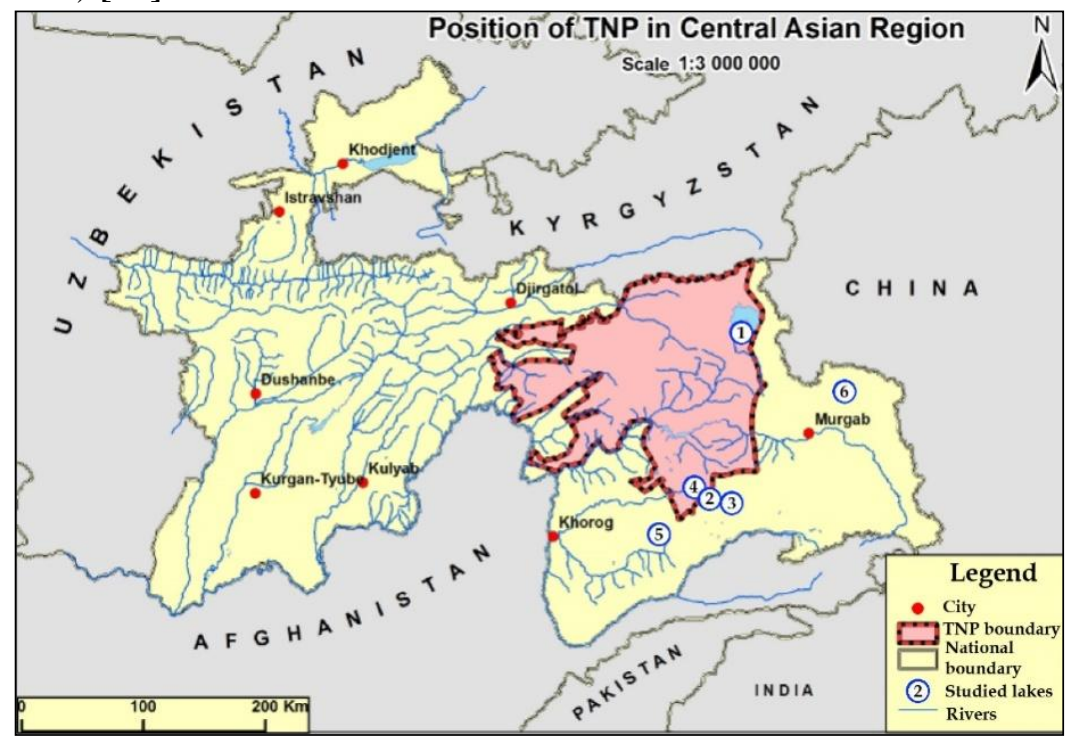

Fig 1. Map of the Tajik National Park and location of studied lakes in Tajikistan in blue circles: 1-Karakul; 2 Bulunkul; 3 -Sassykkul; 4 - Yashilkul; 5 - Turumtaykul; 6-Rangkul

Table 1. Hydrochemical properties of protected lakes in Pamir Mountains according A.M. Muzafarov [10]

\begin{tabular}{|l|l|l|l|}
\hline Formation & Facies & Average Water TDS, $\mathrm{mg} \mathrm{l}^{-1}$ & Lakes \\
\hline Hydrocarbonate & $\mathrm{HCO}_{3}{ }^{-}-\mathrm{Ca}^{2+}-\mathrm{SO}_{4}{ }^{2-}$ & $80-200$ & Yashilkul, Sarezskoye, \\
\hline Hydrocarbonate & $\mathrm{HCO}_{3}{ }^{-}-\mathrm{Ca}^{2+}-\mathrm{Na}^{+}+\mathrm{K}^{+}$ & $30-80$ & Zorkul \\
\hline Hydrocarbonate & $\mathrm{HCO}_{3}{ }^{-}-\mathrm{Na}^{+}+\mathrm{K}^{+}-\mathrm{SO}_{4}{ }^{2-}$ & $150-150$ & Bulunkul, Kichikkul \\
\hline Hydrocarbonate & $\mathrm{HCO}_{3}{ }^{-}-\mathrm{SO}_{4}{ }^{2-}-\mathrm{Ca}^{2+}$ & $200-500$ & Rangkul, Yashilkul, Sarezskoye \\
\hline Sulfate & $\mathrm{SO}_{4}{ }^{2-}-\mathrm{HCO}_{3}{ }^{-}-\mathrm{Ca}^{2+}$ & $>500$ & Rangkul \\
\hline Sulfate & $\mathrm{SO}_{4}{ }^{2-}-\mathrm{HCO}_{3}{ }^{-}-\mathrm{Mg}^{2+}$ & $900-1,500$ & Rangkul, Shorkul \\
\hline Sulfate & $\mathrm{SO}_{4}{ }^{2-}-\mathrm{Cl}^{-}-\mathrm{HCO}_{3}^{-}$ & 2,800 & Karakul \\
\hline Sulfate & $\mathrm{SO}_{4}{ }^{2-}-\mathrm{Cl}^{-}-\mathrm{Mg}^{2+}$ & $>1000$ & Shorkul \\
\hline Sulfate & $\mathrm{SO}_{4}{ }^{2-}-\mathrm{Cl}^{-}-\mathrm{Mg}^{2+}$ & $4500-7600$ & Karakul \\
\hline Sulfate & $\mathrm{SO}_{4}{ }^{2-}-\mathrm{Cl}^{-}-\mathrm{Na}^{+}+\mathrm{K}^{+}$ & $>9,500$ & Karakul \\
\hline Sodium & $\mathrm{Na}^{+}+\mathrm{K}^{+}-\mathrm{SO}_{4}{ }^{2-}-\mathrm{Cl}^{-}$ & $70,000-100,000$ & Sassykkul \\
\hline
\end{tabular}

\subsection{Sampling and laboratory studies}

Earlier was presented total list of diatom algae of high mountains of Tajikistan that has been published in the base of references and our investigation during 2000-2015 [4]. Now the list from which we take in account the lakes floras was enriched by our field trips in 2015-2018 results which were focused on algal communities of six Pamir lakes, especially.

So, the material for this analysis is represented by our own data from 466 samples collected during nine field trips in the summer period of 2000-2018 from six lakes located at an altitude from 3,729 to 4,213 $\mathrm{m}$ above the sea level (Fig. 2, Tab. 2). Algal periphytonic samples were collected by scratching, planktonic samples - by scooping and lather filtering with pore $0.8-1.2 \mathrm{mkm}$. Samples were fixed in $3 \%$ neutral formaldehyde solution, transported to the lab in the icebox. Diatom samples were processed in the laboratory of flora and systematics of thalloid plants of the Institute of Botany, Plant Physiology and Genetics of the Academy of Sciences of the Republic of Tatarstan, the Institute of Evolution of the University of Haifa, and in the Laboratory of Plant Systematics and Geography named I.D. Papanin, RAS in Borok. A light microscope Nikon Eclipse E 600 and a scanning electron microscope JSM-25S were used. For the determination of diatom species, the relevant handbooks, systematic reports, monographs, and individual articles were used [11-14].

The list of revealed diatoms in six lakes was updated with an algaebase.org [15]. Calculation of similarity was doing in the GRAPHS program [16] and network analyses in JASP on the bootnet package in R [17]. The network graphs and Pearson correlation coefficients that JASP produces are based on the R Statistica package [17]. 

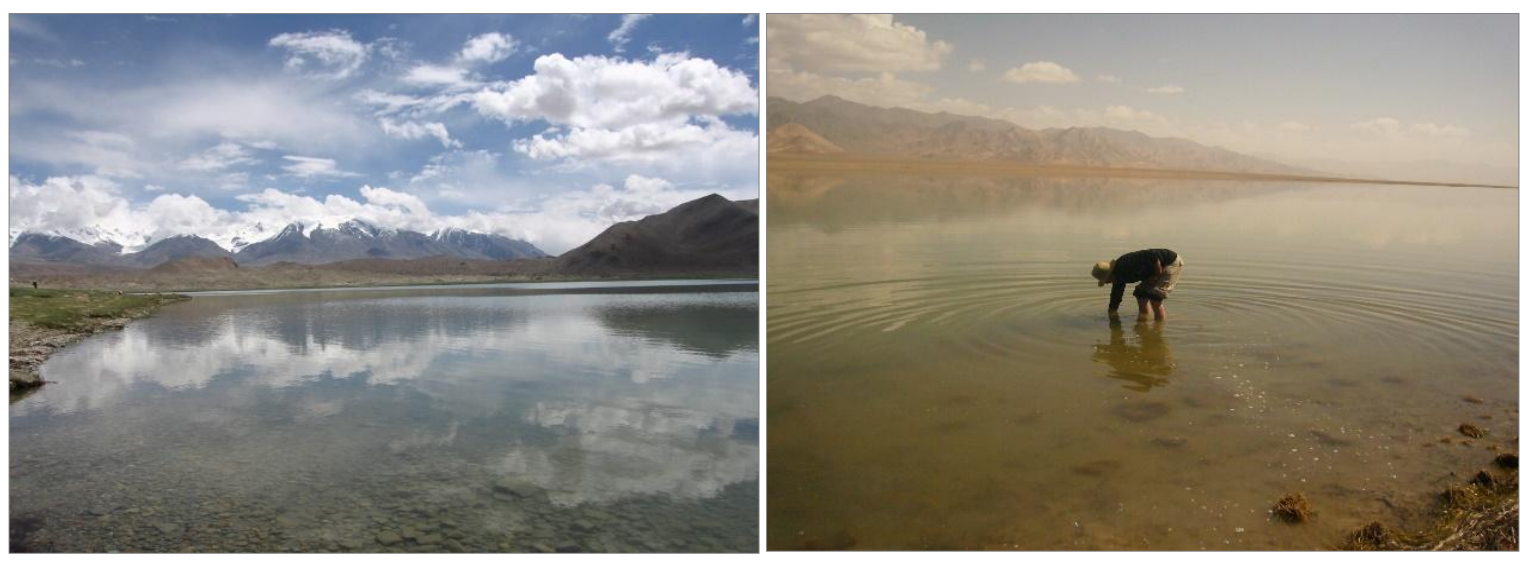

Fig 2. Studied lakes in Pamir Mountains: Lake Karakul (left), Lake Rangkul (right)

\section{RESULTS AND DISCUSSION}

\subsection{Environmental variables}

Data about studied communities from six lakes in Pamir are represented in Table 2. Can be seen that altitude of studied lakes is very high but varied in small amplitude. Nevertheless the water in all six lakes have different salt content and related to three different groups as Hydro carbonate, Sulfate, and Sodium (Table 1), the most varied properties is water TDS. So, whereas TDS in Bulunkul, Yashilkul, Turumtaykul, and Rangkul is about $300 \mathrm{mg} \mathrm{l}^{-1}$, two other lakes have very high level of TDS as 10,867 $\mathrm{mg}^{-1}$ in Karakul and 141,000 $\mathrm{mg}^{-1}$ in Sassykkul.

Table 2. Environmental variables of the studied lakes in the Pamir Mountains

\begin{tabular}{|c|c|c|c|c|c|c|}
\hline $\begin{array}{c}\text { Name of } \\
\text { lake }\end{array}$ & Karakul & Bulunkul & Sassykkul & Yashilkul & Turumtaykul & Rangkul \\
\hline $\begin{array}{c}\text { No. on the } \\
\text { map }\end{array}$ & 1 & 2 & 3 & 4 & 5 & 6 \\
\hline Latitude, N & $\begin{array}{c}39^{\circ} 00^{\prime} \\
33.52^{\prime \prime}\end{array}$ & $\begin{array}{c}37^{\circ} 43^{\prime} \\
28.14^{\prime \prime}\end{array}$ & $\begin{array}{c}37^{\circ} 41^{\prime \prime} \\
48.85^{\prime \prime}\end{array}$ & $37^{\circ} 44^{\prime} 53.42^{\prime \prime}$ & $\begin{array}{c}37^{\circ} 28^{\prime} \\
47.94^{\prime \prime}\end{array}$ & $38^{\circ} 28^{\prime} 32.72^{\prime \prime}$ \\
\hline Longitude, E & $\begin{array}{c}73^{\circ} 33^{\prime} \\
04.93^{\prime \prime}\end{array}$ & $\begin{array}{c}72^{\circ} 57^{\prime} \\
26.67^{\prime \prime}\end{array}$ & $\begin{array}{c}73^{\circ} 10^{\prime} \\
48.48^{\prime \prime}\end{array}$ & $72^{\circ} 54^{\prime} 19.80^{\prime \prime}$ & $\begin{array}{c}72^{\circ} 32^{\prime} \\
42.00^{\prime \prime}\end{array}$ & $74^{\circ} 15^{\prime} 20.39^{\prime \prime}$ \\
\hline Altitude, m & 3921 & 3792 & 3851 & 3729 & 4213 & 3827 \\
\hline $\begin{array}{c}\text { Average } \\
\text { Water TDS, } \\
\text { mg l } 1^{-1}\end{array}$ & $\begin{array}{c}10,867- \\
11,568\end{array}$ & 114.3 & 141,000 & 341.2 & $100-300$ & 463 \\
\hline
\end{tabular}

\subsection{Diversity and ecology of algae and cyanobacteria}

Taxonomical diversity of diatom communities of the studied lakes is presented in Table 3. Altogether 300 species of diatom algae (330 with infraspecific variables) from 76 genera were revealed in six studied Pamir lakes (Table 4).

Table 3. Taxonomical diversity of diatom algae in the Pamir Mountains lakes. Note: Number of lakes like in Table 2: 1 - Karakul; 2 - Bulunkul; 3 - Sassykkul; 4 - Yashilkul; 5 - Turumtaykul; 6 - Rangkul.

\begin{tabular}{|l|l|l|l|l|l|l|}
\hline \multicolumn{1}{|c|}{ Taxa } & $\mathbf{1}$ & $\mathbf{2}$ & $\mathbf{3}$ & $\mathbf{4}$ & $\mathbf{5}$ & $\mathbf{6}$ \\
\hline Achnanthes brevipes C.Agardh var. brevipes & 1 & 0 & 1 & 0 & 0 & 1 \\
\hline Achnanthes brevipes var. intermedia (Kützing) Cleve & 0 & 0 & 1 & 0 & 0 & 0 \\
\hline Achnanthes coarctata (Brébisson ex W.Smith) Grunow & 1 & 0 & 0 & 1 & 0 & 1 \\
\hline Achnanthes dispar var. angustissima (Jasnitsky) Sheshukova & 0 & 0 & 0 & 0 & 1 & 0 \\
\hline Achnanthes pamirensis Hustedt & 0 & 1 & 0 & 0 & 0 & 0 \\
\hline Achnanthidium exiguum (Grunow) Czarnecki & 0 & 0 & 0 & 0 & 1 & 1 \\
\hline Achnanthidium exile (Kützing) Heiberg & 0 & 0 & 0 & 1 & 0 & 0 \\
\hline Achnanthidium lanceolatum var. ventricosum (Hustedt) Poretsky & 1 & 0 & 0 & 0 & 0 & 0 \\
\hline Achnanthidium minutissimum (Kützing) Czarnecki & 1 & 1 & 1 & 1 & 1 & 0 \\
\hline Amphora commutata Grunow & 1 & 1 & 1 & 1 & 1 & 0 \\
\hline Amphora mongolica Østrup var. mongolica & 0 & 0 & 0 & 0 & 1 & 1 \\
\hline
\end{tabular}




\begin{tabular}{|c|c|c|c|c|c|c|}
\hline Amphora mongolica var. cornuta Skvortzov & 1 & 1 & 1 & 1 & 1 & 1 \\
\hline Amphora ovalis (Kützing) Kützing var. ovalis & 1 & 1 & 1 & 1 & 1 & 1 \\
\hline Amphora ovalis var. gracilis (Ehrenberg) Van Heurck & 1 & 1 & 1 & 1 & 1 & 0 \\
\hline Amphora pediculus (Kützing) Grunow in A.W.F.Schmidt & 1 & 0 & 1 & 0 & 0 & 0 \\
\hline Amphora proteus $\mathrm{W}$. Gregory & 0 & 0 & 1 & 0 & 1 & 1 \\
\hline Amphora robusta W.Gregory & 1 & 0 & 1 & 0 & 0 & 1 \\
\hline Aneumastus apiculatus (Østrup) Lange-Bertalot in Lange-Bertalot \& Genkal & 1 & 0 & 0 & 0 & 0 & 0 \\
\hline Aneumastus minor Lange-Bertalot & 1 & 0 & 1 & 0 & 0 & 0 \\
\hline Aneumastus rostratus (Hustedt) Lange-Bertalot & 1 & 1 & 1 & 1 & 1 & 0 \\
\hline $\begin{array}{l}\text { Aneumastus tuscula (Ehrenberg) D.G.Mann \& A.J.Stickle in Round, } \\
\text { R.M.Crawford \& D.G.Mann }\end{array}$ & 1 & 0 & 1 & 1 & 0 & 1 \\
\hline Anomoeoneis costata (Kützing) Hustedt & 1 & 1 & 1 & 1 & 0 & 0 \\
\hline Anomoeoneis sphaerophora Pfitzer var. sphaerophora & 1 & 1 & 1 & 1 & 1 & 1 \\
\hline Anomoeoneis sphaerophora var. guentheri Otto Müller & 0 & 0 & 0 & 0 & 0 & 1 \\
\hline Anomoeoneis sphaerophora var. sculpta (Ehrenberg) Otto Müller & 0 & 0 & 0 & 0 & 0 & 1 \\
\hline Aulacoseira granulata (Ehrenberg) Simonsen & 1 & 1 & 1 & 1 & 1 & 1 \\
\hline Brachysira exilis (Kützing) Round \& D.G.Mann & 1 & 1 & 0 & 1 & 0 & 1 \\
\hline Brachysira microcephala (Grunow) Compère & 1 & 1 & 1 & 1 & 1 & 0 \\
\hline Caloneis bacillum (Grunow) Cleve & 1 & 1 & 0 & 1 & 1 & 1 \\
\hline Caloneis limosa (Kützing) R.M.Patrick & 1 & 1 & 0 & 1 & 1 & 1 \\
\hline Caloneis molaris (Grunow) Krammer in Krammer \& Lange-Bertalot & 0 & 1 & 1 & 1 & 0 & 0 \\
\hline Caloneis nubicola (Grunow) Cleve & 1 & 1 & 1 & 1 & 1 & 0 \\
\hline Caloneis schumanniana (Grunow) Cleve & 1 & 1 & 1 & 1 & 1 & 0 \\
\hline Caloneis silicula (Ehrenberg) Cleve var. silicula & 1 & 1 & 1 & 1 & 1 & 0 \\
\hline Caloneis silicula var. jenissejensis Grunow & 1 & 0 & 0 & 0 & 0 & 0 \\
\hline Caloneis silicula var. kjellmaniana (Cleve) Cleve & 1 & 1 & 1 & 1 & 1 & 0 \\
\hline Caloneis tenuis (Gregory) Krammer & 0 & 0 & 0 & 0 & 1 & 1 \\
\hline Caloneis undulata (Gregory) Krammer & 0 & 0 & 0 & 0 & 1 & 1 \\
\hline Caloneis ventricosa (Ehrenberg) F. Meister var. ventricosa & 1 & 0 & 0 & 0 & 1 & 1 \\
\hline Caloneis ventricosa var. truncatula (Grunow) Meister & 1 & 1 & 1 & 1 & 1 & 1 \\
\hline $\begin{array}{l}\text { Cavinula cocconeiformis (W.Gregory ex Greville) D.G.Mann \& A.J.Stickle } \\
\text { in Round, R.M.Crawford \& D.G.Mann }\end{array}$ & 0 & 0 & 1 & 0 & 0 & 1 \\
\hline $\begin{array}{l}\text { Cavinula scutelloides (W.Smith) Lange-Bertalot in Lange-Bertalot \& } \\
\text { Metzeltin }\end{array}$ & 1 & 1 & 1 & 1 & 1 & 0 \\
\hline Cocconeis disculus (Schumann) Cleve in Cleve \& Jentzsch & 0 & 0 & 1 & 0 & 0 & 0 \\
\hline Cocconeis fluviatilis JHWallace & 0 & 0 & 0 & 0 & 0 & 1 \\
\hline Cocconeis lineata Ehrenberg & 0 & 0 & 0 & 0 & 0 & 1 \\
\hline Cocconeis pediculus Ehrenberg & 0 & 0 & 0 & 0 & 0 & 1 \\
\hline Cocconeis placentula Ehrenberg var. placentula & 1 & 1 & 0 & 1 & 0 & 1 \\
\hline Cocconeis placentula var. euglypta (Ehrenberg) Grunow & 0 & 0 & 0 & 0 & 0 & 1 \\
\hline $\begin{array}{l}\text { Cocconeis placentula var. intermedia (Héribaud-Joseph \& M.Peragallo) } \\
\text { Cleve }\end{array}$ & 1 & 1 & 0 & 1 & 1 & 0 \\
\hline Cocconeis scutellum Ehrenberg & 1 & 1 & 1 & 1 & 1 & 0 \\
\hline Craticula ambigua (Ehrenberg) D.G.Mann in Round, Crawford \& D.G.Mann & 0 & 0 & 0 & 0 & 1 & 1 \\
\hline Craticula cuspidata (Kützing) Mann & 0 & 0 & 0 & 0 & 1 & 1 \\
\hline $\begin{array}{l}\text { Craticula halophila (Grunow) D.G.Mann in Round, R.M.Crawford \& } \\
\text { D.G.Mann var. halophila }\end{array}$ & 1 & 1 & 1 & 1 & 0 & 0 \\
\hline Craticula halophila var. subcapitata (Østrup) Czarnecki & 1 & 1 & 1 & 1 & 0 & 0 \\
\hline Ctenophora pulchella (Ralfs ex Kützing) D.M. Williams et Round & 1 & 1 & 1 & 0 & 0 & 1 \\
\hline Cyclostephanos mansfeldensis Houk, Kleen \& H.Tanaka & 1 & 1 & 1 & 1 & 0 & 1 \\
\hline Cyclotella caspia Grunow & 1 & 1 & 1 & 1 & 1 & 1 \\
\hline Cyclotella meneghiniana Kützing & 0 & 0 & 0 & 0 & 1 & 1 \\
\hline Cymatopleura elliptica (Brébisson) W.Smith & 0 & 0 & 0 & 0 & 1 & 1 \\
\hline Cymatopleura librile (Ehrenberg) Pantocsek & 1 & 1 & 0 & 1 & 1 & 1 \\
\hline Cymbella affinis Kützing & 0 & 1 & 0 & 1 & 0 & 1 \\
\hline
\end{tabular}




\begin{tabular}{|c|c|c|c|c|c|c|}
\hline Cymbella angustata var. diversistriata Muzafarov & 0 & 1 & 0 & 1 & 0 & 0 \\
\hline Cymbella aspera (Ehrenberg) Cleve var. aspera & 1 & 0 & 1 & 0 & 0 & 1 \\
\hline Cymbella aspera var. intermedia Skvortzov & 1 & 1 & 1 & 0 & 0 & 1 \\
\hline Cymbella cistula (Ehrenberg) O.Kirchner & 1 & 1 & 1 & 1 & 1 & 0 \\
\hline Cymbella helvetica Kützing & 1 & 1 & 1 & 0 & 1 & 1 \\
\hline Cymbella hustedtii f. lineolata Muzafarov & 1 & 0 & 0 & 0 & 0 & 1 \\
\hline Cymbella hustedtii Krasske f. hustedtii & 1 & 1 & 1 & 1 & 1 & 1 \\
\hline Cymbella laevis Nägeli in Rabenhorst & 1 & 1 & 0 & 1 & 0 & 1 \\
\hline Cymbella lanceolata var. cornuta f. minuta Muzafarov & 0 & 0 & 0 & 0 & 0 & 1 \\
\hline Cymbella obtusiuscula Kützing & 1 & 1 & 1 & 1 & 1 & 0 \\
\hline Cymbella parva (W.Smith) Kirchner & 0 & 1 & 1 & 1 & 0 & 0 \\
\hline Cymbella proschkinae Muzafarov & 0 & 0 & 0 & 0 & 0 & 1 \\
\hline Cymbella stuxbergii (Cleve) Cleve & 0 & 0 & 0 & 0 & 0 & 1 \\
\hline Cymbella subsymmetrica J.B.Petersen & 0 & 1 & 0 & 1 & 0 & 0 \\
\hline Cymbella tartuensis Molder & 0 & 0 & 1 & 0 & 1 & 0 \\
\hline Cymbopleura amphicephala (Nägeli) Krammer & 0 & 1 & 1 & 1 & 0 & 1 \\
\hline Cymbopleura anglica (Lagerstedt) Krammer & 0 & 0 & 0 & 0 & 1 & 0 \\
\hline Cymbopleura angustata (W. Smith) Krammer & 1 & 0 & 1 & 0 & 1 & 1 \\
\hline Cymbopleura florentina (Grunow) Krammer & 0 & 0 & 0 & 0 & 0 & 1 \\
\hline Cymbopleura gutwinskii (Wislouch) Krammer & 0 & 1 & 1 & 1 & 0 & 0 \\
\hline Cymbopleura inaequalis (Ehrenberg) Krammer & 0 & 1 & 1 & 1 & 1 & 0 \\
\hline Cymbopleura incerta (Grunow) Krammer & 0 & 1 & 1 & 1 & 0 & 0 \\
\hline Cymbopleura reinhardtii (Grunow) Krammer & 1 & 0 & 0 & 0 & 1 & 0 \\
\hline Cymbopleura subaequalis (Grunov) Krammer & 0 & 0 & 0 & 0 & 0 & 1 \\
\hline Delicata delicatula (Kützing) Krammer & 0 & 0 & 0 & 0 & 1 & 1 \\
\hline Denticula elegans Kützing & 0 & 0 & 0 & 0 & 0 & 1 \\
\hline Denticula kuetzingii Grunow & 0 & 0 & 0 & 0 & 0 & 1 \\
\hline Denticula tenuis Kützing & 0 & 1 & 0 & 1 & 1 & 0 \\
\hline Diadesmis contenta var. biceps (Grunow) P.B.Hamilton & 0 & 0 & 0 & 0 & 0 & 1 \\
\hline Diatoma elongata (Lyngbye) C.Agardh & 1 & 1 & 1 & 1 & 1 & 1 \\
\hline Diatoma tenuis Agardh & 0 & 0 & 0 & 0 & 0 & 1 \\
\hline Diatoma vulgaris Bory var. vulgaris & 1 & 1 & 1 & 1 & 1 & 0 \\
\hline Diatoma vulgaris var. producta Grunow & 1 & 0 & 0 & 0 & 0 & 0 \\
\hline Didymosphenia geminata (Lyngbye) Mart.Schmidt & 1 & 1 & 1 & 1 & 1 & 1 \\
\hline Diploneis elliptica (Kützing) Cleve & 0 & 0 & 0 & 0 & 0 & 1 \\
\hline Diploneis oculata (Brébisson) Cleve & 0 & 0 & 0 & 0 & 0 & 1 \\
\hline Diploneis ovalis (Hilse) Cleve & 0 & 0 & 0 & 0 & 0 & 1 \\
\hline Diploneis parma Cleve & 0 & 0 & 0 & 0 & 0 & 1 \\
\hline Diploneis subovalis Cleve & 1 & 1 & 1 & 1 & 1 & 0 \\
\hline Encyonema elginense (Krammer) D.G.Mann in Round, Crawford \& Mann & 0 & 1 & 1 & 0 & 1 & 1 \\
\hline Encyonema gracile Rabenhorst & 1 & 0 & 1 & 0 & 1 & 1 \\
\hline Encyonema lacustre (C.Agardh) Pantocsek & 1 & 1 & 1 & 1 & 1 & 0 \\
\hline $\begin{array}{l}\text { Encyonema leibleinii (C.Agardh) W.J.Silva, R.Jahn, T.A.Veiga Ludwig \& } \\
\text { M.Menezes }\end{array}$ & 0 & 0 & 0 & 0 & 1 & 1 \\
\hline Encyonema minutum (Hilse in Rabenhorst) D.G. Mann & 0 & 0 & 0 & 0 & 1 & 0 \\
\hline Encyonema silesiacum (Bleisch in Rabenhorst) D.G. Mann & 0 & 0 & 0 & 0 & 1 & 0 \\
\hline Encyonema ventricosum (C.Agardh) Grunow & 1 & 1 & 1 & 0 & 0 & 0 \\
\hline Encyonopsis falaisensis (Grunow) Krammer & 1 & 1 & 1 & 0 & 0 & 1 \\
\hline Encyonopsis microcephala (Grunow) Krammer & 0 & 0 & 0 & 0 & 1 & 1 \\
\hline Entomoneis alata (Ehrenberg) Ehrenberg & 1 & 1 & 1 & 1 & 1 & 0 \\
\hline Entomoneis ornata (Bailey) Reimer in R.M.Patrick \& C.W.Reimer & 1 & 1 & 1 & 1 & 1 & 0 \\
\hline Entomoneis paludosa (W. Smith) Reimer var. paludosa & 1 & 1 & 0 & 0 & 1 & 1 \\
\hline Entomoneis paludosa var. duplex (Donkin) Makarova \& Achmetova & 1 & 0 & 0 & 0 & 1 & 1 \\
\hline Epithemia adnata (Kützing) Brébisson var. & 1 & 1 & 1 & 0 & 0 & 1 \\
\hline
\end{tabular}




\begin{tabular}{|c|c|c|c|c|c|c|}
\hline Epithemia adnata var. saxonica (Kützing) R.M.Patrick in Patrick \& Reimer & 1 & 1 & 0 & 1 & 0 & \\
\hline Epithemia argus var. longicornis (Ehrenberg) Grunow & 0 & 0 & 0 & 0 & 0 & 1 \\
\hline Epithemia argus (Ehrenberg) Kützing var. argus & 1 & 1 & 1 & 1 & 1 & 1 \\
\hline Epithemia argus var. alpestris (W.Smith) Grunow & 1 & 1 & 1 & 1 & 1 & 1 \\
\hline Epithemia operculata (C.Agardh) Ruck \& Nakov in Ruck et al. & 1 & 1 & 0 & 1 & 1 & 0 \\
\hline Epithemia parallela (Grunow) Ruck \& Nakov in Ruck et al. & 0 & 1 & 0 & 1 & 1 & 0 \\
\hline Epithemia porcellus Kützing & 1 & 0 & 1 & 0 & 0 & 0 \\
\hline Epithemia sorex Kützing & 1 & 1 & 1 & 1 & 1 & 1 \\
\hline Epithemia turgida (Ehrenberg) Kützing & 0 & 0 & 0 & 0 & 1 & 1 \\
\hline Eucocconeis flexella (Kützing) Meister & 0 & 1 & 0 & 1 & 1 & 0 \\
\hline Eucocconeis quadratarea (Østrup) Lange-Bertalot \& Genkal & 0 & 0 & 0 & 0 & 0 & 1 \\
\hline Eunotia arcus Ehrenberg & 0 & 0 & 0 & 0 & 1 & 1 \\
\hline Eunotia bidens Ehrenberg & 0 & 1 & 1 & 1 & 0 & 0 \\
\hline Eunotia exigua (Brébisson in Kützing) Rabenhorst & 0 & 1 & 1 & 1 & 1 & 0 \\
\hline Eunotia lunaris (Ehrenberg) Grunow & 0 & 0 & 0 & 1 & 0 & 0 \\
\hline Eunotia minor (Kützing) Grunow & 0 & 0 & 0 & 1 & 0 & 0 \\
\hline Eunotia polydentula (Brun) Husted & 0 & 0 & 0 & 1 & 0 & 1 \\
\hline Eunotia praerupta Ehrenberg & 0 & 0 & 0 & 1 & 0 & 0 \\
\hline Fallacia pygmaea (Kützing) De Mann & 0 & 0 & 0 & 0 & 1 & 1 \\
\hline Fragilaria acus (Kützing) Lange-Bertalot in Krammer \& Lange-Bertalot & 0 & 0 & 0 & 0 & 1 & 1 \\
\hline Fragilaria alpestris Krasske ex Hustedt & 0 & 0 & 0 & 0 & 1 & 1 \\
\hline $\begin{array}{l}\text { Fragilaria amphicephaloides Lange-Bertalot in Hofmann, Werum \& Lange- } \\
\text { Bertalot }\end{array}$ & 0 & 0 & 1 & 1 & 1 & 0 \\
\hline Fragilaria bidens Heiberg & 0 & 0 & 0 & 0 & 1 & 0 \\
\hline Fragilaria capucina Desmazières var. capucina & 0 & 0 & 0 & 1 & 1 & 0 \\
\hline Fragilaria capucina var. lanceolata Grunow in van Heurck & 0 & 0 & 1 & 0 & 0 & 0 \\
\hline Fragilaria crotonensis Kitton & 0 & 1 & 1 & 1 & 1 & 0 \\
\hline Fragilaria inflata var. istvanffyi (Pantoscek) Hustedt & 0 & 0 & 1 & 0 & 0 & 0 \\
\hline Fragilaria radians (Kützing) D.M.Williams \& Round & 0 & 0 & 0 & 0 & 0 & 1 \\
\hline $\begin{array}{l}\text { Fragilaria recapitellata Lange-Bertalot \& Metzeltin in Metzeltin, Lange- } \\
\text { Bertalot \& Nergui }\end{array}$ & 0 & 0 & 0 & 1 & 0 & 0 \\
\hline Fragilaria vaucheriae (Kützing) J.B.Petersen & 1 & 0 & 1 & 1 & 1 & 1 \\
\hline Fragilariforma bicapitata (A.Mayer) D.M.Williams \& Round & 0 & 0 & 0 & 0 & 1 & 1 \\
\hline Fragilariopsis cylindrus (Grunow) Helmcke \& Krieger & 0 & 0 & 0 & 0 & 0 & 1 \\
\hline Frustulia rhomboides (Ehrenberg) De Toni & 0 & 0 & 0 & 0 & 1 & 0 \\
\hline $\begin{array}{l}\text { Genkalia digituloides (Lange-Bertalot) Lange-Bertalot \& Kulikovskiy in } \\
\text { Kulikovskiy et al. }\end{array}$ & 1 & 1 & 1 & 1 & 1 & 1 \\
\hline Gomphoneis herculeana (Ehrenberg) Cleve & 0 & 0 & 0 & 0 & 0 & 1 \\
\hline Gomphonema acuminatum Ehrenberg & 0 & 1 & 0 & 0 & 0 & 1 \\
\hline Gomphonema angustatum (Kützing) Rabenhorst & 1 & 1 & 1 & 1 & 1 & 1 \\
\hline Gomphonema constrictum var. capitatum (Ehrenberg) Grunow & 1 & 1 & 0 & 0 & 0 & 0 \\
\hline Gomphonema gracile Ehrenberg & 1 & 1 & 1 & 1 & 0 & 1 \\
\hline Gomphonema grunowii R.M.Patrick \& Reimer & 1 & 1 & 1 & 1 & 1 & 1 \\
\hline Gomphonema kobayasii Kociolek \& J.C.Kingston & 0 & 0 & 0 & 0 & 0 & 1 \\
\hline Gomphonema lagenula Kützing & 0 & 1 & 1 & 1 & 1 & 0 \\
\hline Gomphonema longiceps Ehrenberg & 1 & 1 & 1 & 1 & 1 & 0 \\
\hline Gomphonema micropus Kützing & 0 & 0 & 0 & 0 & 1 & 1 \\
\hline Gomphonema olivaceum (Hornemann) Brébisson & 1 & 0 & 0 & 0 & 0 & 0 \\
\hline Gomphonema parvulum (Kützing) Kützing & 1 & 1 & 1 & 1 & 1 & 0 \\
\hline Gomphonema subclavatum (Grunow) Grunow & 1 & 1 & 1 & 1 & 1 & 0 \\
\hline Gomphonema truncatum Ehrenberg & 1 & 1 & 1 & 1 & 1 & 0 \\
\hline Gomphosinica hedinii (Hustedt) Kociolek, Q.-M.You, Q.-X.Wang \& Q.Liu & 1 & 1 & 1 & 1 & 1 & 1 \\
\hline Gyrosigma acuminatum (Kützing) Rabenhorst & 0 & 1 & 1 & 1 & 0 & 0 \\
\hline Gyrosigma attenuatum (Kützing) Rabenhorst & 0 & 0 & 0 & 1 & 1 & 1 \\
\hline Gyrosigma peisonis (Grunow) Hustedt в Pascher & 0 & 0 & 0 & 1 & 1 & 1 \\
\hline
\end{tabular}




\begin{tabular}{|c|c|c|c|c|c|c|}
\hline Halamphora acutiuscula (Kützing) Levkov & 0 & 0 & 0 & 0 & 1 & 1 \\
\hline Halamphora coffeiformis (C.Agardh) Levkov & 1 & 1 & 1 & 0 & 0 & 1 \\
\hline Halamphora latecostata J.G.Stepanek \& Kociolek & 0 & 0 & 0 & 0 & 0 & 1 \\
\hline Halamphora normanii (Rabenhorst) Levkov & 1 & 1 & 1 & 1 & 1 & 0 \\
\hline $\begin{array}{l}\text { Halamphora perpusilla (Grunow) Q.-M.You \& Kociolek в Q.-M.You, } \\
\text { JPKociolek \& W.Wang }\end{array}$ & 1 & 1 & 1 & 1 & 1 & 0 \\
\hline Halamphora subcapitata (Kisselew) Levkov & 1 & 0 & 1 & 0 & 1 & 0 \\
\hline Halamphora veneta (Kützing) Levkov & 1 & 1 & 1 & 1 & 1 & 0 \\
\hline Hannaea arcus (Ehrenberg) RMPatrick в RMPatrick \& CWReimer & 1 & 1 & 1 & 1 & 1 & 1 \\
\hline Hannaea arcus var. amphioxys (Rabenhorst) R.M.Patrick & 1 & 1 & 1 & 1 & 0 & 0 \\
\hline Hantzschia abundans Lange-Bertalot & 0 & 0 & 0 & 0 & 1 & 0 \\
\hline $\begin{array}{l}\text { Hantzschia amphioxys (Ehrenberg) Grunow in Cleve \& Grunow var. } \\
\text { amphioxys }\end{array}$ & 1 & 1 & 1 & 1 & 1 & 0 \\
\hline Hantzschia amphioxys var. vivax (Hantzsch) Grunow in Cleve \& Grunow & 0 & 1 & 0 & 1 & 0 & 0 \\
\hline Hantzschia compacta (Hustedt) Lange-Bertalot in Lange-Bertalot \& Genkal & 0 & 0 & 0 & 0 & 0 & 1 \\
\hline Hippodonta capitata (Ehrenberg) Lange-Bertalot, Metzeltin \& Witkowski & 0 & 0 & 0 & 0 & 0 & 1 \\
\hline Hippodonta hungarica (Grunow) Lange-Bertalot, Metzeltin \& Witkowski & 1 & 1 & 1 & 0 & 1 & 0 \\
\hline $\begin{array}{l}\text { Humidophila contenta (Grunow) Lowe, Kociolek, J.R.Johansen, Van de } \\
\text { Vijver, Lange-Bertalot \& Kopalová }\end{array}$ & 0 & 0 & 0 & 0 & 0 & 1 \\
\hline Iconella curvula (W.Smith) Ruck \& Nakov in Ruck et al. & 1 & 0 & 0 & 1 & 1 & 0 \\
\hline Iconella linearis (W.Smith) Ruck \& Nakov in Ruck et al. & 1 & 1 & 1 & 1 & 1 & 1 \\
\hline Iconella tenera (W.Gregory) Ruck \& Nakov in Ruck et al. & 1 & 1 & 0 & 0 & 1 & 0 \\
\hline Kobayasiella subtilissima (Cleve) Lange-Bertalot & 1 & 0 & 1 & 0 & 0 & 0 \\
\hline Kurtkrammeria aequalis (W.Smith) L.Bahls & 1 & 0 & 1 & 1 & 0 & 0 \\
\hline Lindavia antiqua (W.Smith) Nakov, Guillory, Julius, Theriot \& Alverson & 1 & 1 & 1 & 1 & 0 & 1 \\
\hline $\begin{array}{l}\text { Lindavia bodanica (Eulenstein ex Grunow) T.Nakov, Guillory, Julius, } \\
\text { Theriot \& Alverson }\end{array}$ & 1 & 1 & 1 & 1 & 0 & 0 \\
\hline Lindavia comta (Kützing) Nakov, Gullory, Julius, Theriot \& Alverson & 0 & 1 & 1 & 1 & 1 & 1 \\
\hline Lindavia kuetzingiana var. radiosa (Fricke) T.Nakov et al. & 0 & 0 & 0 & 0 & 1 & 0 \\
\hline Lindavia lacunarum (Hustedt) Nakov, Guillory, Julius, Theriot \& Alverson & 1 & 1 & 0 & 0 & 0 & 1 \\
\hline Luticola mutica (Kützing) D.G.Mann in Round, R.M.Crawford \& D.G.Mann & 0 & 0 & 0 & 0 & 1 & 0 \\
\hline $\begin{array}{l}\text { Luticola nivalis (Ehrenberg) D.G.Mann in Round, R.M.Crawford \& } \\
\text { D.G.Mann } 1990\end{array}$ & 0 & 0 & 0 & 0 & 1 & 1 \\
\hline $\begin{array}{l}\text { Mastogloia albertii A.Pavlov, E.Jovanovska, C.E.Wetzel, L.Ector \& } \\
\text { Z.Levkov }\end{array}$ & 0 & 0 & 0 & 0 & 1 & 1 \\
\hline Mastogloia baltica Grunow in Van Heurck & 1 & 1 & 1 & 1 & 1 & 0 \\
\hline Mastogloia braunii Grunow & 1 & 1 & 1 & 1 & 1 & 0 \\
\hline Mastogloia elliptica (C.Agardh) Cleve in A.W.F.Schmidt & 1 & 0 & 0 & 0 & 0 & 0 \\
\hline $\begin{array}{l}\text { Mastogloia pseudosmithii S.S.Lee, E.E.Gaiser, B.Van de Vijver, } \\
\text { M.B.Edlund \& Spaulding }\end{array}$ & 0 & 0 & 0 & 0 & 0 & 1 \\
\hline Mastogloia smithii Thwaites ex W.Smith & 0 & 0 & 0 & 0 & 1 & 1 \\
\hline Meridion circulare (Greville) C.Agardh & 1 & 1 & 1 & 1 & 1 & 1 \\
\hline Meridion constrictum Ralfs & 0 & 0 & 0 & 0 & 0 & 1 \\
\hline Navicula capitatoradiata H.Germain & 0 & 0 & 0 & 0 & 0 & 1 \\
\hline Navicula cari Ehrenberg & 0 & 0 & 0 & 0 & 0 & 1 \\
\hline Navicula cincta (Ehrenberg) Ralfs & 0 & 1 & 1 & 1 & 1 & 0 \\
\hline Navicula cryptocephala Kützing var. cryptocephala & 0 & 1 & 0 & 0 & 1 & 1 \\
\hline Navicula cryptocephala var. lata Poretz. et Anissimova & 0 & 0 & 0 & 0 & 1 & 1 \\
\hline Navicula dicephala Ehrenberg & 1 & 1 & 1 & 0 & 0 & 1 \\
\hline Navicula lacustris var. parallela Wisl. \& Kolbe & 1 & 0 & 0 & 0 & 0 & 0 \\
\hline Navicula lanceolata Ehrenberg & 1 & 1 & 1 & 1 & 1 & 0 \\
\hline Navicula libonensis Schoeman & 1 & 0 & 0 & 0 & 0 & 0 \\
\hline Navicula minima Grunow in Van Heurck & 0 & 0 & 0 & 0 & 0 & 1 \\
\hline Navicula oblonga (Kützing) Kützing & 1 & 1 & 1 & 1 & 1 & 1 \\
\hline Navicula peregrina (Ehrenberg) Kützing & 1 & 1 & 1 & 1 & 1 & 0 \\
\hline Navicula radiosa Kützing & 0 & 0 & 0 & 0 & 0 & 1 \\
\hline
\end{tabular}




\begin{tabular}{|c|c|c|c|c|c|c|}
\hline Navicula rhynchocephala Kützing & 0 & 1 & 0 & 1 & 1 & 0 \\
\hline Navicula rostellata Kützing & 1 & 1 & 1 & 1 & 1 & 0 \\
\hline Navicula rotaeana (Rabenhorst) Grunow in van Heurck & 0 & 0 & 0 & 0 & 1 & 1 \\
\hline Navicula salinarum f. minima Kolbe & 0 & 0 & 0 & 0 & 1 & 1 \\
\hline Navicula semen Ehrenberg & 1 & 0 & 0 & 0 & 0 & 1 \\
\hline Navicula tenella Brébisson ex Kützing & 0 & 1 & 0 & 1 & 1 & 0 \\
\hline Navicula tuscula f. intermedia Kisselev & 1 & 1 & 1 & 1 & 0 & 0 \\
\hline Navicula viridula (Kützing) Ehrenberg & 1 & 0 & 0 & 0 & 1 & 1 \\
\hline Navicula vulpina Kützing & 1 & 1 & 1 & 1 & 1 & 0 \\
\hline Navicula semen Ehrenberg & 0 & 0 & 0 & 0 & 1 & 0 \\
\hline Navicymbula pusilla (Grunow in A. Schmidt) Krammer & 0 & 0 & 0 & 0 & 1 & 0 \\
\hline Neidiomorpha binodis (Ehrenberg) M.Cantonati, Lange-Bertalot \& N.Angeli & 0 & 1 & 0 & 0 & 0 & 0 \\
\hline Neidium iridis (Ehrenberg) Cleve & 0 & 1 & 1 & 1 & 1 & 1 \\
\hline Neidium kozlowii Mereschkovsky & 1 & 0 & 1 & 1 & 0 & 0 \\
\hline Nitzschia amphibia Grunow var. amphibia & 0 & 0 & 0 & 0 & 0 & 1 \\
\hline Nitzschia amphibia var. thermalis Grunow & 0 & 1 & 0 & 1 & 1 & 0 \\
\hline Nitzschia communis Rabenhorst & 1 & 1 & 1 & 1 & 1 & 0 \\
\hline Nitzschia denticula Grunow in Cleve \& Grunow & 0 & 0 & 0 & 0 & 0 & 1 \\
\hline Nitzschia dissipata (Kützing) Rabenhorst & 0 & 0 & 1 & 0 & 1 & 0 \\
\hline Nitzschia distans $\mathrm{W}$.Gregory & 1 & 0 & 1 & 0 & 0 & 0 \\
\hline Nitzschia fonticola (Grunow) Grunow in Van Heurck & 0 & 0 & 0 & 0 & 0 & 1 \\
\hline Nitzschia frustulum (Kützing) Grunow in Cleve \& Grunow var. frustulum & 0 & 0 & 0 & 0 & 0 & 1 \\
\hline Nitzschia frustulum var. subsalina Hustedt & 1 & 1 & 1 & 0 & 1 & 0 \\
\hline Nitzschia frustulum var. perpusilla (Rabenhorst) Van Heurck & 0 & 0 & 0 & 0 & 0 & 1 \\
\hline Nitzschia gracilis Hantzsch & 0 & 0 & 0 & 0 & 0 & 1 \\
\hline Nitzschia heidenii (Meister) Hustedt & 0 & 0 & 0 & 0 & 0 & 1 \\
\hline Nitzschia holsatica Hustedt in A.W.F.Schimidt & 1 & 1 & 1 & 0 & 0 & 0 \\
\hline Nitzschia hungarica var. pantocsekii Wislouch \& Poretsky & 1 & 0 & 0 & 1 & 0 & 0 \\
\hline Nitzschia kuetzingiana Hilse & 0 & 0 & 0 & 0 & 0 & 1 \\
\hline Nitzschia linearis W.Smith & 0 & 0 & 0 & 0 & 0 & 1 \\
\hline Nitzschia obtusa W.Smith & 0 & 0 & 0 & 0 & 0 & 1 \\
\hline Nitzschia ostenfeldii Hustedt & 0 & 0 & 0 & 0 & 0 & 1 \\
\hline Nitzschia palea (Kützing) W.Smith & 0 & 0 & 0 & 0 & 0 & 1 \\
\hline Nitzschia paleacea (Grunow) Grunow in Van Heurck & 0 & 0 & 0 & 0 & 1 & 1 \\
\hline Nitzschia pusilla Grunow & 0 & 1 & 0 & 0 & 0 & 0 \\
\hline Nitzschia regula Hustedt & 0 & 0 & 0 & 0 & 1 & 0 \\
\hline Nitzschia sigma (Kützing) W.Smith & 0 & 0 & 0 & 0 & 1 & 0 \\
\hline Nitzschia sigmoidea (Nitzsch) W.Smith & 1 & 1 & 1 & 1 & 0 & 0 \\
\hline Odontidium hyemale (Roth) Kützing & 1 & 0 & 1 & 0 & 1 & 1 \\
\hline Pantocsekiella kuetzingiana (Thwaites) KTKiss \& E.Ács in Ács et al. & 1 & 1 & 1 & 1 & 1 & 1 \\
\hline $\begin{array}{l}\text { Paraplaconeis compositestriata (Jasnitsky) Kulikovskiy, Metzeltin \& Lange- } \\
\text { Bertalot in Kulikovskiy et al. }\end{array}$ & 1 & 0 & 1 & 1 & 1 & 1 \\
\hline $\begin{array}{l}\text { Paraplaconeis subplacentula (Hustedt) Kulikovskiy \& Lange-Bertalot in } \\
\text { Kulikovskiy et al. }\end{array}$ & 0 & 0 & 1 & 0 & 0 & 1 \\
\hline Pinnularia appendiculata (C.Agardh) Schaarschmidt & 0 & 0 & 0 & 0 & 0 & 1 \\
\hline $\begin{array}{l}\text { Pinnularia biundulata (Otto Müller) Kulikovskiy \& Genkal in Kulikovskiy, } \\
\text { Genkal \& Mikheeva }\end{array}$ & 1 & 0 & 1 & 0 & 0 & 1 \\
\hline Pinnularia borealis Ehrenberg & 0 & 0 & 0 & 0 & 1 & 1 \\
\hline Pinnularia boyeri RMPatrick & 0 & 1 & 1 & 1 & 0 & 0 \\
\hline Pinnularia brauniana (Grunow) Studnicka & 1 & 1 & 1 & 1 & 1 & 0 \\
\hline Pinnularia brebissonii (Kützing) Rabenhorst & 0 & 0 & 0 & 0 & 1 & 1 \\
\hline Pinnularia brevicostata Cleve & 1 & 1 & 1 & 1 & 1 & 0 \\
\hline Pinnularia canadodivergens Kulikovskiy, Lange-Bertalot \& Metzeltin & 0 & 0 & 0 & 0 & 1 & 0 \\
\hline Pinnularia divergentissima (Grunow) Cleve & 0 & 1 & 0 & 1 & 0 & 0 \\
\hline Pinnularia fonticola Hustedt & 1 & 1 & 1 & 1 & 1 & 0 \\
\hline
\end{tabular}




\begin{tabular}{|c|c|c|c|c|c|c|}
\hline Pinnularia globiceps W.Gregory & 0 & 0 & 1 & 0 & 1 & 1 \\
\hline Pinnularia gracillima W.Gregory & 0 & 0 & 0 & 0 & 1 & 1 \\
\hline Pinnularia grunowii Krammer & 0 & 0 & 0 & 0 & 1 & 0 \\
\hline Pinnularia lata (Brébisson) W.Smith var. lata & 1 & 1 & 1 & 1 & 1 & 0 \\
\hline Pinnularia lata var. minor (Grunow) Cleve & 1 & 1 & 1 & 1 & 1 & 0 \\
\hline Pinnularia microstauron (Ehrenberg) Cleve & 1 & 0 & 1 & 0 & 1 & 1 \\
\hline Pinnularia rangoonensis Grunow ex Cleve & 0 & 0 & 0 & 0 & 1 & 1 \\
\hline Pinnularia rhombica Hustedt in Schmidt et al. & 0 & 0 & 0 & 0 & 1 & 1 \\
\hline Pinnularia schoenfelderi Krammer & 0 & 0 & 0 & 0 & 1 & 1 \\
\hline Pinnularia septentrionalis K.Krammer & 0 & 0 & 0 & 0 & 1 & 0 \\
\hline Pinnularia socialis var. debesii (Hustedt) Krammer & 1 & 1 & 1 & 1 & 1 & 0 \\
\hline Pinnularia subborealis Hustedt & 1 & 0 & 0 & 1 & 0 & 0 \\
\hline Pinnularia subcapitata $\mathrm{W}$.Gregory & 0 & 0 & 0 & 0 & 1 & 1 \\
\hline Pinnularia subtibetana Muzafarov & 0 & 0 & 0 & 0 & 0 & 1 \\
\hline Pinnularia tabellaria Ehrenberg & 1 & 1 & 1 & 1 & 1 & 0 \\
\hline Pinnularia viridiformis Krammer & 0 & 0 & 0 & 0 & 1 & 0 \\
\hline Pinnularia viridis (Nitzsch) Ehrenberg var. viridis & 1 & 1 & 1 & 1 & 1 & 1 \\
\hline Pinnularia viridis var. diminuta Ant.Mayer & 1 & 0 & 0 & 0 & 0 & 1 \\
\hline Placoneis exigua (W.Gregory) Mereschkovsky & 1 & 1 & 1 & 0 & 0 & 0 \\
\hline Placoneis gastrum (Ehrenberg) Mereschkowsky & 1 & 0 & 0 & 0 & 1 & 1 \\
\hline Planothidium dispar (Cleve) Witkowski, Lange-Bertalot \& Metzeltin & 1 & 1 & 1 & 1 & 1 & 0 \\
\hline Planothidium lanceolatum (Brébisson ex Kützing) Lange-Bertalot & 1 & 1 & 1 & 1 & 1 & 0 \\
\hline Platessa salinarum (Grunow) Lange-Bertalot & 0 & 0 & 0 & 0 & 1 & 1 \\
\hline Prestauroneis integra (W.Smith) Bruder in Bruder \& Medlin & 1 & 0 & 1 & 0 & 0 & 0 \\
\hline Pseudostaurosira brevistriata var. inflata (Pantocsek) M.B.Edlund & 1 & 1 & 1 & 1 & 0 & 0 \\
\hline Pseudostaurosira elliptica (Schumann) Edlund, Morales \& Spaulding & 1 & 0 & 0 & 0 & 0 & 0 \\
\hline Punctastriata lancettula (Schumann) P.B.Hamilton \& P.A.Siver & 1 & 1 & 1 & 1 & 1 & 0 \\
\hline Rhoicosphenia abbreviata (C.Agardh) Lange-Bertalot & 0 & 0 & 0 & 0 & 1 & 1 \\
\hline Rhopalodia gibba (Ehrenberg) Otto Müller & 1 & 1 & 1 & 1 & 1 & 0 \\
\hline Rhopalodia gibberula (Ehrenberg) Otto Müller & 1 & 1 & 0 & 1 & 0 & 1 \\
\hline Sellaphora bacillum (Ehrenberg) D.G. Mann & 0 & 0 & 0 & 0 & 1 & 1 \\
\hline Sellaphora pupula (Kützing) Mereschkovsky & 1 & 0 & 0 & 0 & 1 & 1 \\
\hline Sellaphora rectangularis (W.Gregory) Lange-Bertalot \& Metzeltin & 0 & 0 & 0 & 0 & 1 & 0 \\
\hline Sellaphora tridentula (Krasske) CEWetzel in Wetzel et al. & 1 & 1 & 1 & 1 & 0 & 0 \\
\hline Stauroneis acuta W.Smith & 0 & 0 & 0 & 0 & 1 & 1 \\
\hline Stauroneis anceps Ehrenberg & 1 & 0 & 1 & 1 & 1 & 1 \\
\hline Stauroneis borrichii (J.B.Petersen) J.W.G.Lund & 0 & 0 & 0 & 0 & 1 & 1 \\
\hline Stauroneis gracilis Ehrenberg & 0 & 0 & 0 & 0 & 1 & 0 \\
\hline Stauroneis lauenburgiana Hustedt & 0 & 0 & 0 & 0 & 1 & 1 \\
\hline Stauroneis phoenicenteron (Nitzsch) Ehrenberg & 0 & 1 & 1 & 1 & 0 & 0 \\
\hline Stauroneis smithii Grunow & 0 & 0 & 0 & 0 & 0 & 1 \\
\hline Staurosira construens Ehrenberg var. construens & 1 & 1 & 1 & 1 & 0 & 0 \\
\hline Staurosira construens var. exigua (W.Smith) H.Kobayasi in S.Mayama et al. & 1 & 1 & 1 & 1 & 1 & 0 \\
\hline Staurosira leptostauron (Ehrenberg) Kulikovskiy \& Genkal & 1 & 1 & 1 & 1 & 1 & 0 \\
\hline Staurosirella pinnata (Ehrenberg) Williams and Round & 1 & 1 & 1 & 1 & 1 & 1 \\
\hline Stephanodiscus astraea (Ehrenberg) Grunow in Cleve \& Grunow & 1 & 1 & 1 & 1 & 1 & 1 \\
\hline Stephanodiscus minutulus (Kützing) Cleve \& Möller & 1 & 0 & 0 & 0 & 1 & 0 \\
\hline Surirella angusta Kützing & 1 & 1 & 1 & 1 & 1 & 1 \\
\hline Surirella brebissonii Krammer \& Lange-Bertalot & 0 & 0 & 0 & 0 & 0 & 1 \\
\hline Surirella minuta Brébisson in Kützing & 1 & 1 & 1 & 1 & 0 & 1 \\
\hline Surirella ovalis Brébisson & 1 & 1 & 1 & 1 & 1 & 1 \\
\hline Surirella splendida (Ehrenberg) Kützing & 0 & 0 & 0 & 0 & 1 & 0 \\
\hline Synedra pulchella var. macrocephala Grunow & 1 & 1 & 1 & 1 & 0 & 0 \\
\hline Tabellaria fenestrata (Lyngbye) Kützing & 1 & 1 & 0 & 1 & 1 & 1 \\
\hline
\end{tabular}




\begin{tabular}{|l|l|l|l|l|l|l|}
\hline Tabellaria flocculosa (Roth) Kützing & 1 & 1 & 1 & 1 & 1 & 0 \\
\hline Tryblionella angustata W.Smith & 1 & 0 & 0 & 0 & 0 & 1 \\
\hline Tryblionella apiculata W.Gregory & 1 & 0 & 0 & 0 & 0 & 1 \\
\hline Tryblionella hantzschiana Grunow & 1 & 1 & 1 & 0 & 0 & 0 \\
\hline Tryblionella hungarica (Grunow) Frenguelli & 0 & 0 & 0 & 0 & 0 & 1 \\
\hline Tryblionella levidensis W.Smith & 1 & 0 & 0 & 0 & 0 & 0 \\
\hline Ulnaria biceps (Kützing) Compére & 1 & 1 & 1 & 1 & 1 & 0 \\
\hline Ulnaria ulna (Nitzsch) Compére var. ulna & 0 & 1 & 1 & 1 & 0 & 0 \\
\hline Ulnaria ulna var. aequalis (Kützing) Aboal in Aboal et al. & 0 & 0 & 0 & 0 & 1 & 0 \\
\hline
\end{tabular}

The first step of floristic analysis was to reveal the fullness of species list for each lake. On the base of revealed diatom species list we constructed the Willis curve to assess how representative the diatoms in the studied lakes. Earlier S. Barinova [18] has been find that Willis curve can be criteria for fullness of species list because follow hyperbolic shape only in well-studied algal floras in Eurasia. The Willis curve of the diatom flora in the studied Pamir lakes was determined to be close to the hyperbolic shape (Fig. 3). This situation shows that we have sufficient diatom species in order to make taxonomic and ecological analysis. Therefore, we can do floristic, taxonomic and ecological analysis for the diatom flora in the Pamir lakes as well as to compare it in future to other high mountain lakes floras.
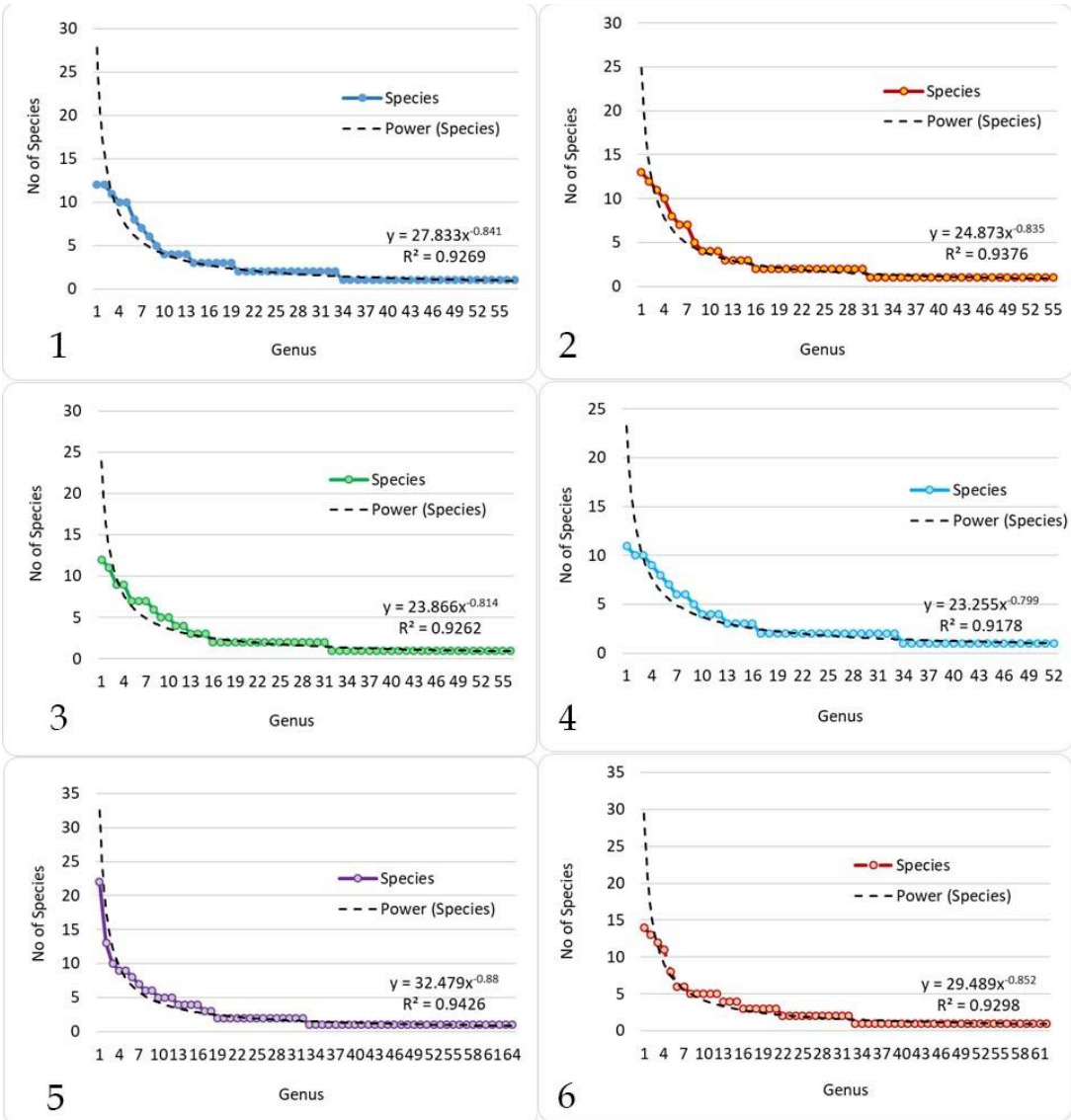

Fig 3. The Willis curve for the diatom flora of the six studied high mountain Pamir lakes shows the number of species per genera (such as 1 genus with 15 species, 2 genera with 6 species, etc.). Number of lakes is the same as figure number and like in Table 2: 1 - Karakul; 2 - Bulunkul; 3 - Sassykkul; 4 - Yashilkul; 5 - Turumtaykul; 6 - Rangkul.

Table 4. Taxonomic distribution of specific, infraspecific taxa and genera of diatom algae in the protected lakes of Pamir with the Subspecies/Species index of intraspecific variation

\begin{tabular}{|c|c|c|c|c|c|c|c|}
\hline Name of lake & Total & Karakul & Bulunkul & Sassykkul & Yashilkul & Turumtaykul & Rangkul \\
\hline No. of lake & & 1 & 2 & 3 & 4 & 5 & 6 \\
\hline Taxa & 330 & 164 & 151 & 152 & 148 & 184 & 176 \\
\hline Species & 300 & 145 & 137 & 139 & 136 & 171 & 162 \\
\hline No of Genera & 76 & 56 & 55 & 56 & 53 & 60 & 60 \\
\hline
\end{tabular}




\begin{tabular}{|c|c|c|c|c|c|c|c|}
\hline Ssp/Sp Index & 1.10 & 1.13 & 1.10 & 1.09 & 1.09 & 1.08 & 1.09 \\
\hline STDEV for genera & 5.51 & 2.75 & 2.74 & 2.50 & 2.44 & 3.45 & 2.96 \\
\hline $\begin{array}{c}\text { Number of genera } \\
\text { over STDEV }\end{array}$ & 17 & 18 & 15 & 15 & 16 & 15 & 21 \\
\hline $\begin{array}{c}\text { No of taxa above } \\
\text { STDEV }\end{array}$ & 207 & 107 & 94 & 93 & 92 & 114 & 122 \\
\hline $\begin{array}{l}\text { Percent of taxa in } \\
\text { abundant genera }\end{array}$ & 62.7 & 66.5 & 63.5 & 66.9 & 63.9 & 66.7 & 71.7 \\
\hline $\begin{array}{c}\text { No of taxa in } 10 \\
\text { genera }\end{array}$ & 159 & 81 & 78 & 74 & 72 & 97 & 88 \\
\hline $\begin{array}{c}\text { Percent of taxa in } 10 \\
\text { genera }\end{array}$ & 48.2 & 50.3 & 52.7 & 53.2 & 50.0 & 56.7 & 51.8 \\
\hline Taxa per genera & 4.3 & 2.9 & 2.7 & 2.5 & 2.7 & 2.9 & 2.8 \\
\hline $\begin{array}{c}\text { Taxa per abundant } \\
\text { genera }\end{array}$ & 12.2 & 5.9 & 6.3 & 6.2 & 5.8 & 7.6 & 5.8 \\
\hline Taxa per 10 genera & 15.9 & 8.1 & 7.8 & 7.4 & 7.2 & 9.7 & 8.8 \\
\hline
\end{tabular}

Table 4. Show that richest communities were found in two freshwater lakes Turumtaykul with 171 species (184 taxa) and Rangkul with 162 species (176 taxa). All other floras are represented of about 150 species in average. The Turumtaykul and Rangkul communities have the same proportion in genera level with 60 against 55 genera average in other lakes. Pearson correlation coefficients were rather weak and insignificant for environmental and biological variables of aquatic ecosystem of six studied lakes represented in Tables 2 and 4 and it is not enough for assessment of speciesenvironment interaction. We compare distribution of species richness over altitude of studied lakes (Fig. 4) and revealed increasing of taxa number with altitude. The same distribution of the lakes water TDS shows oppositional relationship (Fig. 4), so that allow us to conclude that water salinity is more fresh in low altitude lakes and salinity can be named as regulating factor of diatom diversity development in high mountain lakes.

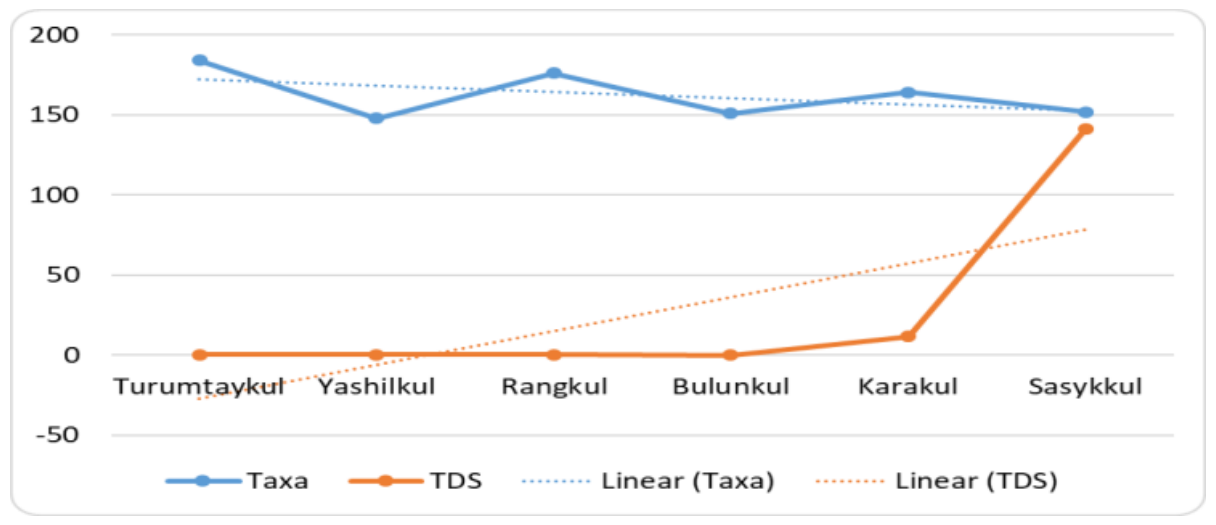

Fig 4. Distribution of the diatom taxa number and water TDS in studied Pamir lakes

Table 5 show generic saturation of each flora of six high mountain lakes. In the top of the table can be seen the richest genera. It was very interesting that Pinnularia was the richest genera in total list as well as in five from six lakes floras. We decide to characterize the head part of each lake flora with different criteria [19]. In Table 4 are represented calculations of criteria for different ways assessment $[20,21]$. Therefore, standard deviation for each distribution of species richness is varied in average about 5.51 that cut off about 17 genera in average. However, number of species in richest genera in this calculation is varied from 122 in the Rangkul to 114 in Turumtaykul in freshwater lakes and about 92-107 in other. In any case, the percent of taxa in abundant genera is varied about $62 \%$ average. Another type of abundant taxa calculation is the taxa number in upper ten genera. Therefore, in Table 4 can be seen that total number of taxa in ten abundant genera is 159 , but in each lake flora, this number is between 72 and 88 taxa. Percent of species in richest ten genera is above $50 \%$, both ways of analysis can be representative for diatom algae of six high mountain lakes in Pamir. We calculated number of taxa per genus for each floras and see that it is in average for total list 4.3 but rather lower in each flora, such as 2.5 taxa per genus in most saline lake Sasykkul and up to 2.9 in other. Comparison of representativeness of taxa number in abundant genera show 12.5 in average for total list and 5.8-7.6 for the flora lake. Number of taxa per ten richest genera is demonstrated the same 
proportion as 15.9 for total list average and 7.2-9.7 per ten genera in the floras of the lakes. Therefore, both type of analysis (by standard deviation and by ten richest genera cut off) demonstrated similar results with richest saturation of taxa in genera for flora of most freshwater Turumtaykul Lake. These results look more levelled when compared to the total algal flora of the South Tajik Depression water bodies at an altitude of up to 2500 [20] and, thus, can characterize the peculiar properties of the Pamir algal flora with prevailing of Pinnularia species in water bodies of different salinity at an altitude of more than $3700 \mathrm{~m}$ a.s.l.

Table 5. Generic saturation of diatom flora of the Pamir lakes. The most abundant genera over Standard Deviation of each lake diversity is marked by bold

\begin{tabular}{|c|c|c|c|c|c|c|c|}
\hline Genera & Total & Karakul & Bulunkul & Sasykkul & Yashilkul & Turumtaykul & Rangkul \\
\hline Pinnularia & 28 & 12 & 10 & 12 & 11 & 21 & 14 \\
\hline Nitzschia & 26 & 8 & 7 & 7 & 5 & 8 & 13 \\
\hline Navicula & 24 & 12 & 12 & 9 & 10 & 14 & 12 \\
\hline Cymbella & 17 & 9 & 11 & 0 & 8 & 0 & 11 \\
\hline Gomphonema & 13 & 9 & 10 & 8 & 8 & 8 & 6 \\
\hline Fragilaria & 12 & 2 & 2 & 6 & 6 & 8 & 4 \\
\hline Caloneis & 12 & 9 & 8 & 6 & 8 & 10 & 6 \\
\hline Epithemia & 10 & 7 & 7 & 5 & 6 & 6 & 7 \\
\hline Cymbopleura & 9 & 2 & 4 & 5 & 4 & 4 & 4 \\
\hline Cocconeis & 8 & 3 & 3 & 2 & 3 & 2 & 5 \\
\hline Amphora & 8 & 6 & 4 & 7 & 4 & 6 & 5 \\
\hline Halamphora & 7 & 5 & 4 & 5 & 3 & 5 & 3 \\
\hline Encyonema & 7 & 3 & 3 & 4 & 0 & 0 & 0 \\
\hline Eunotia & 7 & 0 & 2 & 2 & 6 & 2 & 2 \\
\hline Stauroneis & 7 & 1 & 1 & 2 & 2 & 5 & 5 \\
\hline Mastogloia & 6 & 3 & 2 & 2 & 2 & 4 & 3 \\
\hline Lindavia & 6 & 4 & 5 & 4 & 4 & 3 & 4 \\
\hline Achnanthes & 5 & 2 & 1 & 2 & 1 & 1 & 2 \\
\hline Diploneis & 5 & 1 & 1 & 1 & 1 & 1 & 4 \\
\hline Surirella & 5 & 3 & 3 & 3 & 3 & 3 & 4 \\
\hline Hantzschia & 4 & 1 & 2 & 1 & 2 & 2 & 1 \\
\hline Entomoneis & 4 & 4 & 3 & 2 & 2 & 4 & 2 \\
\hline Denticula & 4 & 0 & 1 & 0 & 1 & 1 & 3 \\
\hline Sellaphora & 4 & 2 & 1 & 1 & 1 & 3 & 2 \\
\hline Anomoeoneis & 4 & 2 & 2 & 2 & 2 & 1 & 3 \\
\hline Aneumastus & 4 & 4 & 0 & 0 & 0 & 0 & 0 \\
\hline Tryblionella & 4 & 3 & 0 & 0 & 0 & 0 & 3 \\
\hline Achnanthidium & 4 & 2 & 1 & 1 & 2 & 2 & 1 \\
\hline Diatoma & 4 & 3 & 2 & 2 & 2 & 2 & 2 \\
\hline Craticula & 3 & 1 & 1 & 1 & 1 & 2 & 2 \\
\hline Gyrosigma & 3 & 0 & 1 & 1 & 3 & 2 & 2 \\
\hline Ulnaria & 3 & 1 & 2 & 2 & 2 & 2 & 0 \\
\hline Placoneis & 2 & 2 & 1 & 1 & 0 & 1 & 1 \\
\hline Tabellaria & 2 & 2 & 2 & 1 & 2 & 2 & 1 \\
\hline Stephanodiscus & 2 & 2 & 1 & 1 & 1 & 2 & 1 \\
\hline Brachysira & 2 & 2 & 2 & 1 & 2 & 1 & 1 \\
\hline Staurosira & 2 & 2 & 2 & 2 & 2 & 1 & 0 \\
\hline Rhopalodia & 2 & 2 & 2 & 1 & 2 & 1 & 1 \\
\hline Cyclotella & 2 & 1 & 1 & 1 & 1 & 2 & 2 \\
\hline Cymatopleura & 2 & 0 & 0 & 0 & 0 & 2 & 2 \\
\hline Pseudostaurosira & 2 & 2 & 1 & 1 & 1 & 0 & 0 \\
\hline Hannaea & 2 & 2 & 2 & 2 & 2 & 1 & 1 \\
\hline Planothidium & 2 & 2 & 2 & 2 & 2 & 2 & 0 \\
\hline Paraplaconeis & 2 & 1 & 0 & 2 & 1 & 1 & 2 \\
\hline Eucocconeis & 2 & 0 & 1 & 0 & 1 & 1 & 0 \\
\hline Neidium & 2 & 0 & 1 & 2 & 2 & 1 & 1 \\
\hline Iconella & 2 & 2 & 2 & 1 & 1 & 2 & 1 \\
\hline Hippodonta & 2 & 0 & 0 & 0 & 0 & 0 & 1 \\
\hline Luticola & 2 & 0 & 0 & 0 & 0 & 2 & 0 \\
\hline
\end{tabular}


Comparative Analysis of Diatom Algae Diversity in the Pamir Protected Lakes, Tajikistan

\begin{tabular}{|c|c|c|c|c|c|c|c|}
\hline Cavinula & 2 & 1 & 1 & 2 & 1 & 1 & 1 \\
\hline Meridion & 2 & 1 & 1 & 1 & 1 & 1 & 2 \\
\hline Punctastriata & 1 & 1 & 1 & 1 & 1 & 1 & 0 \\
\hline Kobayasiella & 1 & 1 & 0 & 1 & 0 & 0 & 0 \\
\hline Synedra & 1 & 1 & 1 & 1 & 1 & 0 & 0 \\
\hline Humidophila & 1 & 0 & 0 & 0 & 0 & 0 & 1 \\
\hline Aulacoseira & 1 & 1 & 1 & 1 & 1 & 1 & 1 \\
\hline Staurosirella & 1 & 1 & 1 & 1 & 1 & 1 & 1 \\
\hline Ctenophora & 1 & 1 & 1 & 1 & 0 & 0 & 1 \\
\hline Cyclostephanos & 1 & 1 & 1 & 1 & 1 & 0 & 1 \\
\hline Encyonopsis & 1 & 0 & 0 & 0 & 0 & 1 & 1 \\
\hline Rhoicosphenia & 1 & 0 & 0 & 0 & 0 & 1 & 1 \\
\hline Fragilariforma & 1 & 0 & 0 & 0 & 0 & 1 & 1 \\
\hline Delicata & 1 & 0 & 0 & 0 & 0 & 1 & 1 \\
\hline Gomphosinica & 1 & 1 & 1 & 1 & 1 & 1 & 1 \\
\hline Prestauroneis & 1 & 1 & 0 & 1 & 0 & 0 & 0 \\
\hline Didymosphenia & 1 & 1 & 1 & 1 & 1 & 1 & 1 \\
\hline Platessa & 1 & 0 & 0 & 0 & 0 & 1 & 1 \\
\hline Neidiomorpha & 1 & 0 & 1 & 0 & 0 & 0 & 0 \\
\hline Gomphoneis & 1 & 0 & 0 & 0 & 0 & 0 & 1 \\
\hline Kurtkrammeria & 1 & 1 & 0 & 1 & 1 & 0 & 0 \\
\hline Genkalia & 1 & 1 & 1 & 1 & 1 & 1 & 1 \\
\hline Frustulia & 1 & 0 & 0 & 0 & 0 & 1 & 0 \\
\hline Odontidium & 1 & 1 & 0 & 1 & 0 & 1 & 1 \\
\hline Fallacia & 1 & 0 & 0 & 0 & 0 & 1 & 1 \\
\hline Fragilariopsis & 1 & 0 & 0 & 0 & 0 & 0 & 1 \\
\hline Navicymbula & 1 & 0 & 0 & 0 & 0 & 1 & 0 \\
\hline
\end{tabular}

Next step in floristic analysis of revealed diversity of diatom algae in six lakes of Pamir was comparative floristic. The dendrograms of species list comparison were constructed with Euclidean distance and Ward method for species and genera level. Fig. 5 demonstrated trees of similarity for species (Fig. 5, 1) and for genera (Fig. 5, 2) numbers in floras of studied lakes and it's are very similar with two different clusters each on the level of 50\% similarity. A flora of the lakes Turumtaykul and Rangkul from the red clusters looks like more diverse on the species and genera level. Remarkable that both these lakes are more fresh from six lakes and more species rich.

Dendrites of overlapping of species diversity (Fig. 6,1) help us to mark the Bulunkul Lake diatoms as floristic core on the species level whereas the Yashilkul flora was core on the genera level (Fig. 6, 2). So, on the Fig. 6 the floras of Bulunkul and Yashilkul have the largest percent of overlapping that allow us to assume that in its environmental properties can be something similar. Table 2 show that water of these both lakes corresponds to the same Hydrocarbonate formation of freshwater lakes.

The comparative floristics of the non-diatom algae of the Pamirs [5] separates the group of six studied lakes into a one cluster, and they also form a separate floristic core in the analysis of the species overlap. This indicates not only their peculiarities compared to other water bodies of Pamir, but also their natural status, which indicates a high level of conservation of diversity in the territory of the Tajik National Park and the adjacent high-mountainous areas.

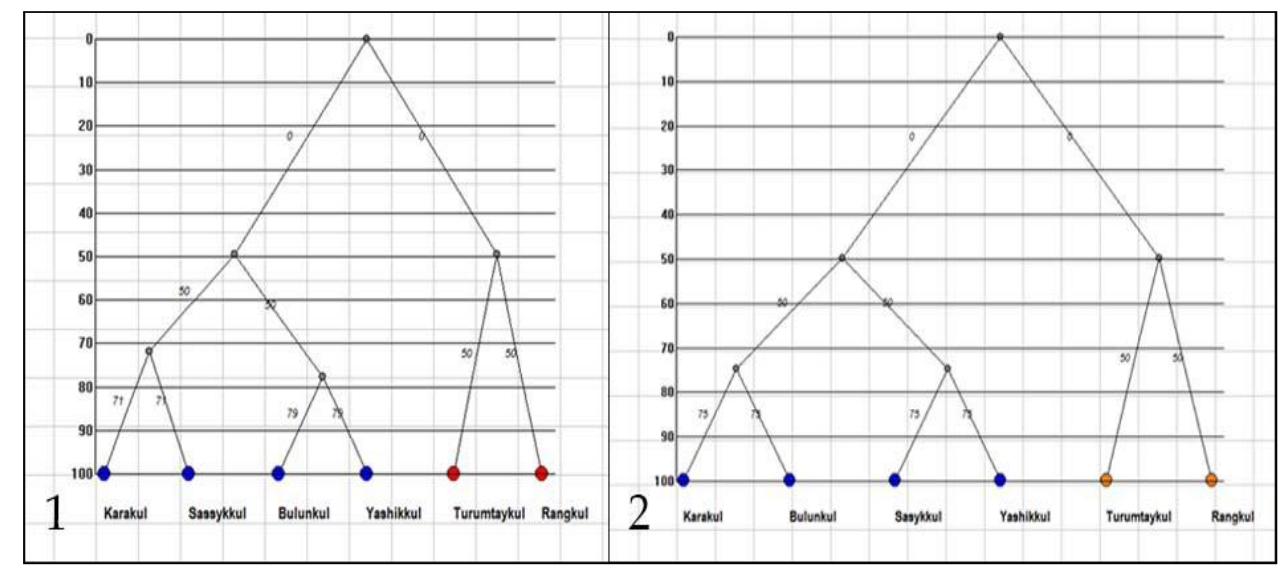

Fig 5. Comparative floristic tree of the diatom taxa number (1) and genera number (2) of six studied Pamir lakes 


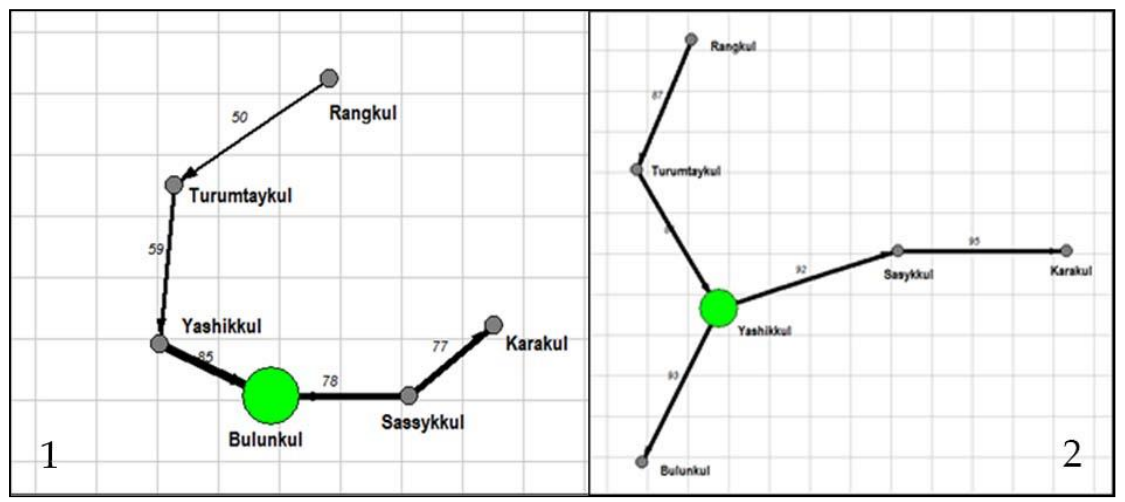

Fig 6. Dendrite of overlapping of the diatom taxa (1) and genera (2) of six studied Pamir lakes. Percent of overlapping is stay in the lines. The line thickness corresponds to overlap percentage

The following step in the analysis of the patterns of distribution of diatom diversity was revealing of correlation between the diatom taxa (Fig. 7, 1) and genera (Fig. 7, 2) in the list of six studied Pamir lakes with the JASP network method. Biological neural networks give a mathematical representation of connections found in ecological, evolutionary, and physiological studies [22]. Between-species interaction networks calculation in biology are in the initial stage today but try to implement the new approach to species interactions is highly concerned with understanding what factors lead to network stability [23]. Can be seen that correlation graphs for species on the base of Table 3 and genera on the base of Table 5 are looks like rather different. This method is implemented in first time for the floristic content correlation. So, the floras of the Bulunkul and Yashilkuk are most similar on the species level. The lakes with high salinity, Sasykkul and Karakul have also similar diatom flora but the Rangkul diatoms are different from each other and demonstrated pecularities. The network graph for correlation on the genera level show different relatedness. Floras of the Bulunkul and Karakul lakes have high level of correlation, then follow Yashilkul and Sasykkul floras, then slightly different were Turumtaykul and Rangkul diatoms. Here we can see only one negative correlation between diatom genera of Yashilkul and Rangkul lakes. Therefore, the stability of studied algal floras in protected high mountain lakes looks like higher on the genera level then on the species level.

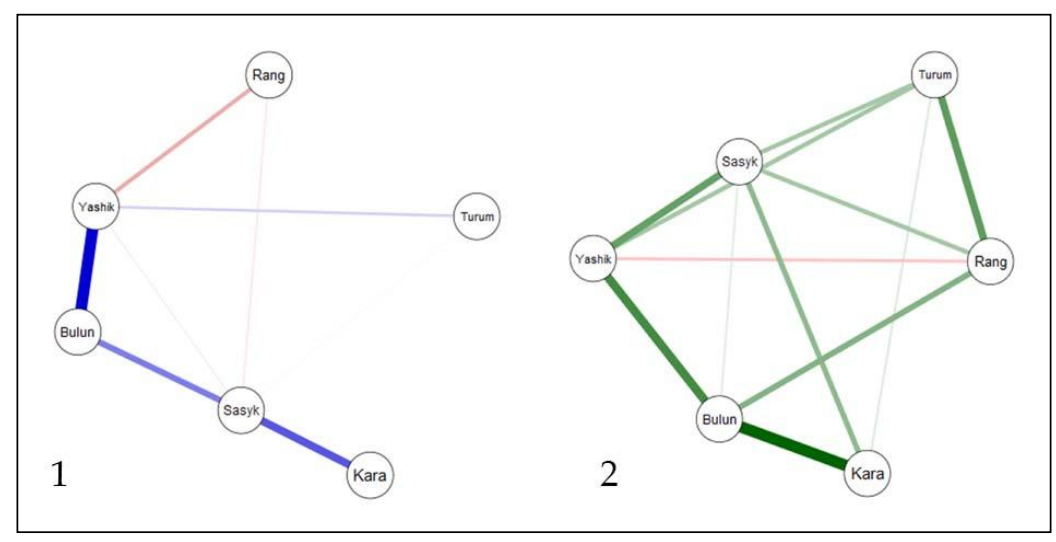

Fig 7. The JASP network graphs of correlation of the diatom taxa (1) and genera (2) of six studied Pamir lakes. The blue lines are positive correlation; the red lines are negative correlation. Line thickness corresponds to the value of the correlation coefficient

Comparison of data about taxonomical diversity of diatom algae that represented in Tables 2, 3, and 4 help us to reveal common distributed and abundant genera in all six lakes as Pinnularia,Nitzschia, Navicula, Gomphonema, Epithemia, and Amphora in the order of decreasing its species number. Genera, which are demonstrated specificity of distribution in studied lakes, were Cymbella, Fragilaria, Cymbopleura, Cocconeis, Eunotia, and Encyonema. Ecologically both groups of species are preferred different environment. Therefore, group of common distributed species preferred wide ecological niche whereas species from second group demonstrated ecological narrow range of environmental variables [21].

We choose one genus from each group: Pinnularia as most abundant in common group, and Cocconeis from second group but represented in each lake communities for clarify environmental 
preferences and compare its distribution to Genera number and Infraspecific variation Ssp/Sp index. Fig. 8 show that generic diversity increase in species rich community of low altitude lakes that similar to $\mathrm{Ssp} / \mathrm{Sp}$ index distribution. It can be interpreted as friendlier environment in relatively low altitudinal lakes. Most abundant and species diverse genus Pinnularia prefer relatively high altitudinal lakes with low water TDS inhabited low diverse communities. Fig. $8(3,4)$ demonstrate different influence of altitude on Pinnularia species distribution that let us to conclude that it not regulated variable. In the same time Cocconeis species abundance and diversity (Fig. 9: 1,2) show lake altitude as regulating factor because in comparison of both graphs these genus members prefer low altitude lakes with low salinity and species rich community.

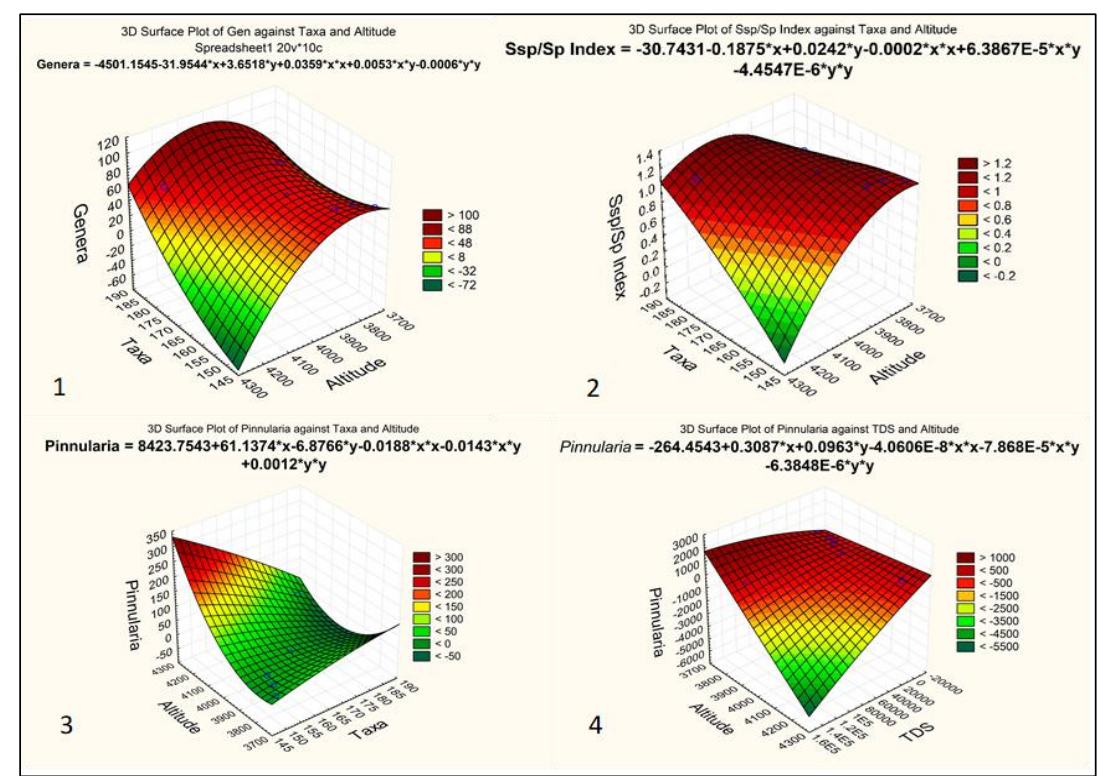

Fig 8. 3D Surface plots of Number of diatom Genera (1), Index of intraspecific variation (2), Pinnularia taxa number (3) over Total taxa number and Altitude of the lake, and Pinnularia taxa number over water TDS and Altitude of the lake (4)

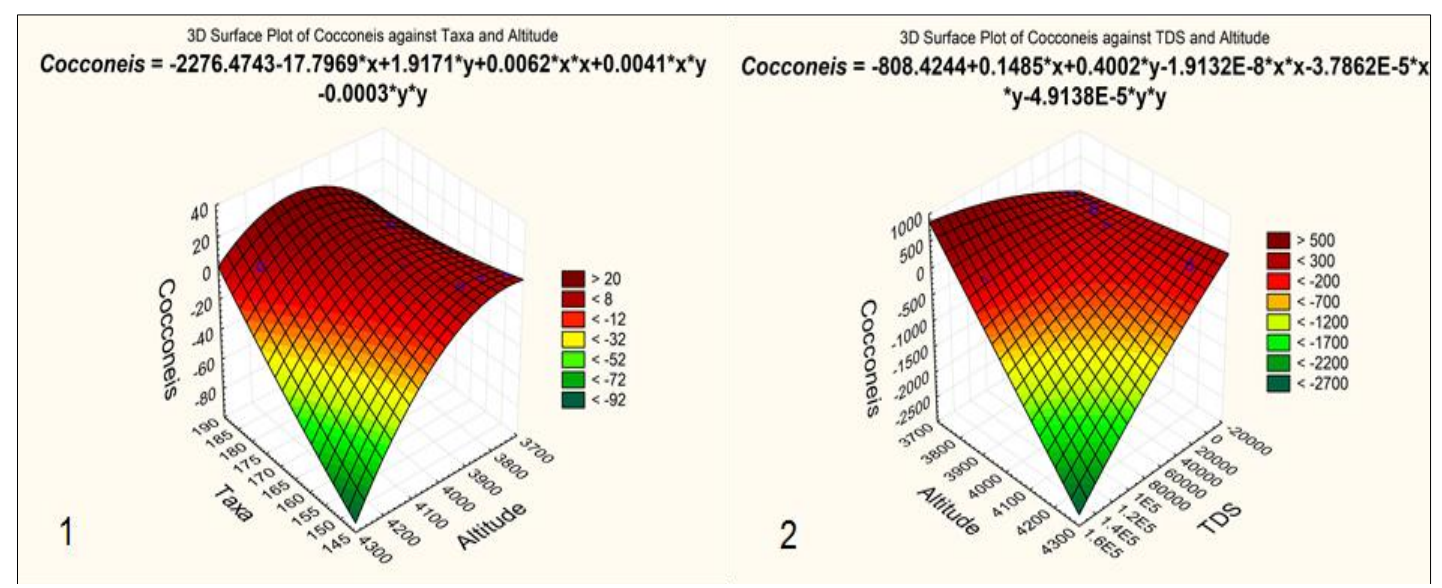

Fig 9. 3D Surface plots of Cocconeis taxa number (1) over Total taxa number and Altitude of the lake, and over water TDS and Altitude of the lake (2)

$\mathrm{Ssp} / \mathrm{Sp}$ index can be a measure of intraspecific variability, which increases under the influence of factors destabilizing the genome of a species. Earlier we revealed altitude as one of the influencing factor on the level of habitats up to 2,600 $\mathrm{m}$ a.s.l. and also high latitude [24,25]. More of them, the index value that we calculated for the Pamir mineral springs algal communities was 1.24. It is sufficiently grater that current calculation and means that water temperature and salts saturation can be influencing factors also. So, we compare distribution on $\mathrm{Ssp} / \mathrm{Sp}$ index value over altitude of the lake and its water TDS (Fig. 10). Can be seen that trend lines of both distributions have weak but positive correlations between index and each environmental factor. Therefore, it allows us to conclude that altitude, high water salinity and temperature are influencing factors, which are destabilizing the genomic stability of algal species and thus can be factors of speciation and evolution of diversity. 


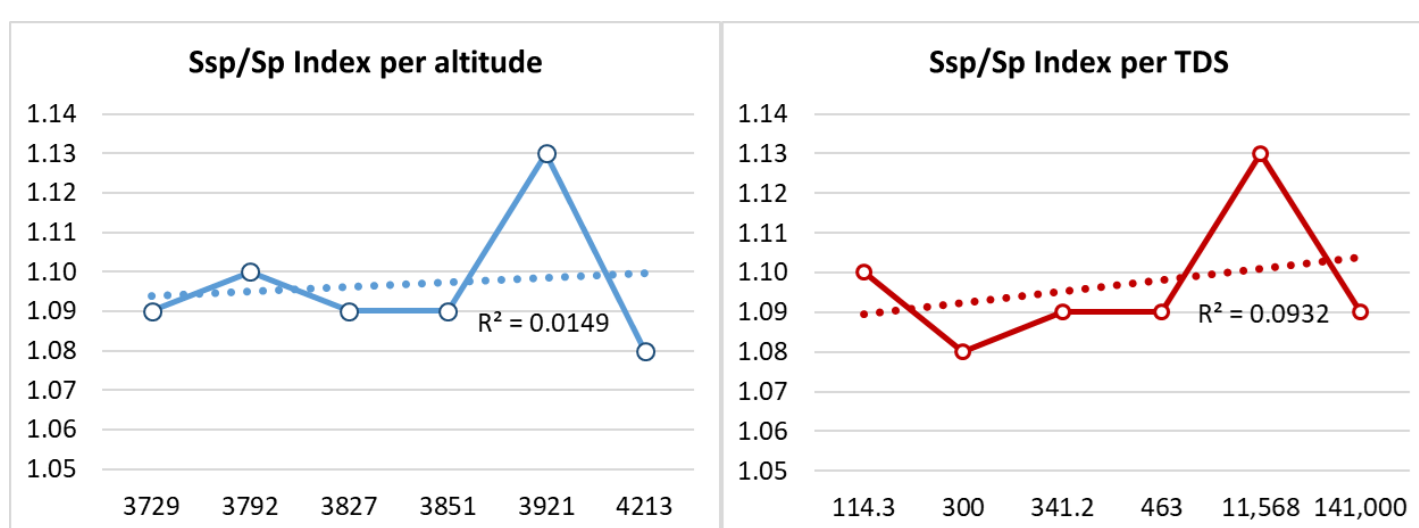

Fig 10. Distribution of Ssp/Sp index of infraspecific variation over lake altitude and water TDS

\section{CONCLUSION}

Altogether 300 species of diatom algae (330 with infraspecific variables) of 76 genera were revealed in 466 samples collected during nine field trips in the summer period of 2000-2018 from six Pamir lakes located at an altitude from 3,729 to 4,213 m above the sea level. Altitude of studied lakes is extremely high but varied in small amplitude. The water in all six lakes have different salt content from $100 \mathrm{mg} \mathrm{l}^{-1}$ in Turumtaykul Lake to $141,000 \mathrm{mg} \mathrm{l}^{-1}$ in Sassykkul. Floristic analysis show that richest communities were found in two freshwater lakes Turumtaykul with 171 species (184 taxa) and Rangkul with 162 species (176 taxa). The water salinity is fresher in low altitude lakes and salinity can be named as regulating factor of diatom diversity development in high mountain lakes as a result of statistical methods implementation. The Pinnularia genus was the richest in total list as well as in five from six lakes floras. Statistical and comparative floristic methods were characterize the peculiar properties of the Pamir algal flora with prevailing of Pinnularia species in water bodies of different salinity at an altitude of more than $3700 \mathrm{~m}$ a.s.l. The separate two floristic core were revealed in the analysis of the species overlapping. This indicates not only lakes flora peculiarities compared to other water bodies of Pamir, but also the natural status of the protected high mountain lakes, that indicates a high level of conservation of diversity in the territory of the Tajik National Park and the adjacent high-mountainous areas. Advanced statistical methods help us to characterize the stability of studied algal floras in protected high mountain lakes as higher on the genera level then on the species level, and therefore species of studied floras can be source of evolutionary speciation.

\section{ACKNOWLEDGMENTS}

This work was partly supported by the Israeli Ministry of Aliyah and Integration.

\section{REFERENCIES}

[1] Pamir, the Columbia Electronic Encyclopedia, Sandbox Networks, Inc., publishing as Infoplease, 24 Jan. 2019, https://www.infoplease.com/encyclopedia/places/asia/south-asia/pamir/, 2019.

[2] Bobohodzhaev I.Ya., Davlatmamadov Sh.M., Healing mineral springs of the Pamir, Dushanbe, 1994.

[3] Tajik National Park (Mountains of the Pamirs), World Heritage List, Available at: http://whc.unesco.org/ en/list/1252/ , 24.01.2019.

[4] Barinova S. and Niyatbekov T. P., Algal diversity of the Pamir high mountain mineral springs in environmental variables gradient, International Journal of Environmental Sciences \& Natural Resources, 7(2), 555706,2017, DOI: 10.19080/IJESNR.2017.07.555706.

[5] Barinova S. and Niyatbekov T., Alpha-biodiversity of nondiatom algae in the Pamir aquatic habitats, Tajikistan, Biodiversity International Journal, 2(3), 236-363,2018, DOI: 10.15406/bij.2018.02.00065.

[6] Niyatbekov T. and Barinova S., Diatom species richness in algal flora of Pamir, Tajikistan, European Scientific Journal, 14(3), 301-323, 2018, Doi: 10.19044/esj.2018.v14n3p301.URL:http://dx.doi.org/ 10.19044/esj.2018.v14n3p301

[7] Niyatbekov T. and Barinova S., Bioindication of water properties by algal communities in the Pamir high mountain mineral and thermal springs, Transylvanian Review of Systematical and Ecological Research, 20(3), "The Wetlands Diversity", 1-30, 2018.

[8] Niyatbekov T. and Barinova S., Bioindication of aquatic habitats with diatom algae in the Pamir Mountains, Tajikistan, MOJ Ecology \& Environmental Sciences, 3(3), 117-120, 2018, https://doi.org/ 10.15406/mojes.2018.03.00075 
[9] Niyatbekov T.P. and Barinova S.S., Diatoms (Bacillariophyta) of the thermal and mineral water sources of Pamir, Biota and Environment, 2, 5-23, 2018. (In Russian).

[10] Muzafarov A. M., Algal flora of the Central Asian water bodies, Uzbekistan Academy of Science Publisher, Tashkent, 1965.

[11] Key to freshwater algae of the USSR, Iss. 4, Diatoms, Soviet science, Moscow, 1951.

[12] Krammer K. and Lange-Bertalot H., Bacillariophyceae, Teil 3: Centrales, Fragilariaceae, Eunotiaceae, Süsswasserflora von Mitteleuropa, 2(3), 1991, Gustav Fisher Verlag, Stuttgart, Germany.

[13] Lange-Bertalot H. and Metzeltin D., Indicators of oligotrophy - 800 taxa representative of three ecologically distinct lake types, Carbonate buffered - Oligodystrophic - Weakly buffered soft water. Lange-Bertalot, H. (ed.), Iconographia Diatomologica, Annotated Diatom Micrographs, Vol. 2, Ecology, Diversity, Taxonomy, Koeltz Scientific Books, Königstein, Germany, 1996.

[14] Genkal C.I., Chekryzheva T.A. and Komulainen S.F., Diatoms of ponds and watercourses of Karelia, Scientific World, Moscow, 2015.

[15] Guiry M.D. and Guiry G.M, Algae Base, World-wide electronic publication, National University of Ireland Press, Galway, http: // www.algaebase.org, 24.01.2019.

[16] Novakovsky A. B., Abilities and base principles of program module "GRAPHS", Komi Scientific Center Publisher, Syktyvkar, Russia, 2004.

[17] Love J., Selker R., Marsman M., Jamil T., Dropmann D., Verhagen A. J., Ly A., Gronau Q. F., Smira M., Epskamp S., Matzke D., Wild A., Rouder J. N., Morey R. D. and Wagenmakers E. J., JASP: graphical statistical software for common statistical designs, J. Stat. Softw., 88(2), 1-17, 2019, doi:10.18637/ jss.v088.i02.

[18] Barinova S., Systemic criteria for the analysis of alpha- and gamma-diversity of freshwater algae, Int. J. Environ. Sci. Nat. Res., 4(2), 555633, 2017, DOI: 10.19080/IJESNR.2017.04.555633.

[19] Jiyenbekov A., Barinova S., Bigaliev A., Nurashov S., Sametova E. and Fahima T., Algal comparative floristic of the Alakol Lake Natural State Reserve and other lakes in Kazakhstan, MOJ Ecology \& Environmental Sciences, 3(4), 252-258, 2018, DOI: 10.15406/mojes.2018.03.00096.

[20] Barinova S.S., and Boboev M.T., Critical approach to the floristic analysis of freshwater algal flora on the example of South-Tajik Depression, Bulletin of Moscow Society of Naturalists. Biology Series, 120(1), 40-48, 2015. (In Russian).

[21] Barinova S. S., Medvedeva L. A. and Anissimova O. V., Diversity of algal indicators in environmental assessment, Pilies Studio, Tel Aviv, 2006. (In Russian).

[22] Proulx S. R., Promislow D. E. L. and Phillips P. C., Network thinking in ecology and evolution, Trends in Ecology and Evolution, 20(6), 345-353, 2005.

[23] Romanuk T.N., Vogt R.J., Young A., Tuck, C. and Carscallen, M.W., Maintenance of positive diversitystability relations along a gradient of environmental stress, PLoS ONE, 5(4), e10378, 2010, https://doi.org/10.1371/journal.pone.0010378.

[24] Barinova S., Boboev M. and Hisoriev H., Freshwater algal diversity of the South-Tajik Depression in a high mountainous extreme environment, Turkish Journal of Botany, 39, 535-546 and Supplement 1-22, 2015.

[25] Barinova S., Gabyshev V., Boboev M., Kukhaleishvili L. and Bilous O., Algal indication of climatic gradients, American Journal of Environmental Protection, Special Issue: Applied Ecology: Problems, Innovations, 4(3-1), 72-77, 2015, doi: 10.11648/j.ajep.s.2015040301.22.

Citation: S. Barinova, T. Niyatbekov. (2019). "Comparative Analysis of Diatom Algae Diversity in the Pamir Protected Lakes, Tajikistan". International Journal of Advanced Research in Botany (IJARB), 5(2), pp.1 -17. DOI: http://dx.doi.org/10.20431/2455-4316.0502001.

Copyright: () 2019 Authors. This is an open-access article distributed under the terms of the Creative Commons Attribution License, which permits unrestricted use, distribution, and reproduction in any medium, provided the original author and source are credited 